University of San Diego

Digital USD

1996-05-01

\title{
The Effect of Written Information and Reassurance on Patient Satisfaction, Anxiety, and Intent to Return for Emergency Care
}

June Andrea DNSc

University of San Diego

Follow this and additional works at: https://digital.sandiego.edu/dissertations

Part of the Nursing Commons

\section{Digital USD Citation}

Andrea, June DNSc, "The Effect of Written Information and Reassurance on Patient Satisfaction, Anxiety, and Intent to Return for Emergency Care" (1996). Dissertations. 254.

https://digital.sandiego.edu/dissertations/254

This Dissertation: Open Access is brought to you for free and open access by the Theses and Dissertations at Digital USD. It has been accepted for inclusion in Dissertations by an authorized administrator of Digital USD. For more information, please contact digital@sandiego.edu. 


\author{
THE EFFECT OF WRITTEN INFORMATION AND REASSURANCE ON \\ PATIENT SATISFACTION, ANXIETY, AND INTENT TO \\ RETURN FOR EMERGENCY CARE \\ by \\ June Andrea \\ A dissertation presented to the \\ FACULTY OF THE PHILIP Y. HAHN SCHOOL OF NURSING \\ UNIVERSITY OF SAN DIEGO \\ In partial fulfillment of the \\ requirements for the degree \\ DOCTOR OF NURSING SCIENCE
}

May 1996

Reproduced with permission of the copyright owner. Further reproduction prohibited without permission. 


\section{ABSTRACT}

Patients' satisfaction is one of the primary goals of emergency department (ED) providers today. As emergency departments are overcrowded, stressful environments, anxious patients want to be kept informed. Nurses have the opportunity to meet these needs and possibly influence the patients' perception of the experience and intent to return for future care. This study examined the effects of providing written information on ED arrival and reassurance at 30-minute intervals on patjent satisfaction, anxiety, and intent to return for emergency care.

The design was a posttest-only design involving a comparison between the control and three experimental. groups. Two hundred and forty patients participated in the study, approximately 60 per group. All subjects were asked to rate their level of anxiety on arrival and discharge from the ED, complete the Consumer Emergency Care Satisfaction Scale, and the Intent to Return scale.

There were no statistically significant differences among the four groups $(\underline{p}<.05)$. Patient satisfaction scores and intent to return scores were high. Anxiety scores were low. Implications and recommendations from this study were made for nursing research, clinical practice, administrative practice, and education. Nurse researchers 
need to conduct qualitative research on patient anxiety in the ED setting. Instruments measuring anxiety and satisfaction in the ED setting need to be developed and refined. Nursing interventions to improve the quality of the ED experience need to be identified and tested. 
Copyright by June Andrea 1996

ALL RIGHTS RESERVED

Reproduced with permission of the copyright owner. Further reproduction prohibited without permission. 


\section{DEDICATION}

This dissertation is dedicated to the patients of Sharp Memorial Hospital who volunteered to participate in the study and to the nursing staff who have provided support and encouragement throughout this endeavor. 


\section{ACKNOWLEDGMENTS}

I want to thank, first and foremost, Marion Hubbard, who provided the scholarship which assisted me through the doctoral program. For her generosity and support of the nursing profession, I am sincerely thankful.

I extend a special thank you to my dissertation committee for their guidance and support. To Dr. Rita Snyder-Halpern, Chairperson, I owe much appreciation for her commitment, encouragement, and expertise. To Dr. Janet Harrison and Dr. John Ronchetto, I thank you for your time, insight, and suggestions which strengthened my work.

There are too many friends to name whose kind words have helped me reach this goal. I thank all of you. I especially thank Rita Cheek for the numerous phone calls during the difficult times and Kimberly Eichler for your friendship. Thanks also to Dan Gross and Maryann Cone for your encouragement and advice. You are both an inspiration to me.

Finally, I thank my family for believing in me. To Tom Melheim, I am forever grateful for your unending love and support. It has been a long and incredible journey. 
TABLE OF CONTENTS

Page

DEDICATION

ACKNOWLEDGMENTS . . . . . . . . . . . . . . . . . .

LIST OF TABLES . . . . . . . . . . . . . . . . . . . . vii vi vi

LIST OF FIGURES . . . . . . . . . . . . . . . . . . v viii

LIST OF APPENDICES . . . . . . . . . . . . . . . . .

CHAPTER

1 INTRODUCTION . . . . . . . . . . . . . . . . 1

Conceptual Framework . . . . . . . . . 6

Purpose of the study . . . . . . . . . 10

Hypotheses . . . . . . . . . . . . . 10

Definition of Terms . . . . . . . . . . 11

Summary . . . . . . . . . . . . . . 12

2 REVIEW OF THE LITERATURE . . . . . . . . . . 14

Satisfaction . . . . . . . . . . . . . 14

Consumer Satisfaction . . . . . . . . 14

Patient Satisfaction with Hospital

Care ............... . . 19

Patient Satisfaction with Emergency

Care . . . . . . . . . . . . . 22

Patient Satisfaction with Nursing

Care .. . . . . . . . . . . . 27

Anxiety . . . . . . . . . . . . . . 31

Intent to Return . . . . . . . . . . . 32

Written Information Intervention . . . . 34 iv 
Reassurance Intervention . . . . . . . . 35

Summary . . . . . . . . . . . . . . . 39

3 METHODOLOGY . . . . . . . . . . . . . . . 40

Design . . . . . . . . . . . . . . . . . 40

Setting . . . . . . . . . . . . . . . 41

Sample . . . . . . . . . . . . . . . 42

Instrumentation . . . . . . . . . . . 48

Consumer Emergency Care Satisfaction
Scale (CECSS) . . . . . . . . . . . . 48

Anxiety . . . . . . . . . . . . . 51

Intent to Return . . . . . . . . . . 52

Demographic Data . . . . . . . . . 53

Data Collection . . . . . . . . . . 53

Pilot Study . . . . . . . . . . . . 53

Data Collection Process . . . . . . . . 55

Study Intervention Protocols . . . . . . 56

Protocol 1 (Control) . . . . . . . . . . 57

Protocol 2 (Written Information) . . . . 58

Protocol 3 (Reassurance) . . . . . . . . 58

Protocol 4 (Combination) . . . . . . . 62

Data Analysis Techniques . . . . . . . . 62

Summary . . . . . . . . . . . . . . . . 64

4 RESULTS AND DISCUSSION . . . . . . . . . . . 66

Data Analysis Related to the Research
Hypotheses . . . . . . . . . . . . 66

Discussion .............. . . . . 71

Randomization . . . . . . . . . . . 72

Patient Satisfaction . . . . . . . . 75 
Anxiety . . . . . . . . . . . . . 78

Intent to Return . . . . . . . . . . . 81

Written Information Intervention . . . . 82

Reassurance Intervention .. . . . . . 83

Summary . . . . . . . . . . . . . . 84

5 SUMMARY AND IMPLICATIONS . . . . . . . . . . 85

Summary . . . . . . . . . . . . . 85

Conceptual Framework . . . . . . . . . . 86

Implications . . . . . . . . . . . . . 87

Nursing Research .. . . . . . . . . 87

Clinical Practice . . . . . . . . . . 89

Administrative Practice . . . . . . . . 90

Nursing Education .. . . . . . . . 90

Conclusions . . . . . . . . . . . . . 91

REFERENCES . . . . . . . . . . . . . . . . . 93 
1. Dimensions of patient Satisfaction by Author

2. Demographics of Subjects by Group . . . . . .

3. Kruskal-Wallis Test for Reassurance Intervention Differences by Group

4. Test for Variable Skewness and Kurtosis . . .

5. Kruskal-Wallis Test for Anxiety-Pre by Group $(\underline{N}=240)$. . . . . . . . . . . .

6. Kruskal-Wallis Test for Anxiety-Post by Group $(\underline{N}=240)$

7. Kruskal-Wallis Test for CECSS by Group $(\underline{N}=239)$. . . . . . . . . . . . . . .

8. Kruskal-wallis Test for Intent to Return by Group $(\underline{\mathrm{N}}=236) \quad$. . . . . . . . . . . .

9. Kruskal-Wallis Test for Gender Differences . . . . . . . . . . . . . . . .

10. Spearman Correlation Coefficients for Age and Dependent Variables 
LIST OF FIGURES

FIGURE

PAGE

1. Patient satisfaction model

viii

Reproduced with permission of the copyright owner. Further reproduction prohibited without permission. 


\section{LIST OF APPENDICES}

APPENDIX

PAGE

A SAMPLE CONSUMER EMERGENCY CARE SATISFACTION SCALE AND SCORING PROCEDURE . . . . . . . . . 105

B LINEAR ANALOGUE ANXIETY SCALE . . . . . . . . 109

C SAMPLE INTENT TO RETURN SCALE ITEMS . . . . . 111

D WRITTEN INFORMATION SHEET . . . . . . . . . . 113

E REASSURANCE DATA COLLECTION FORM . . . . . . 116

F DEMOGRAPHIC DATA COLIECTION FORM . . . . . . 118

G UNIVERSITY OF SAN DIEGO HUMAN SUBJECTS APPROVAL . . . . . . . . . . . . . . . . 120

$\begin{array}{llll}\text { H SHARP HEALTHCARE INSTITUTIONAL REVIEW } & & \\ \text { BOARD APPROVAL TO CONDUCT RESEARCH } & \text {. . . . . } & 122\end{array}$

I PATIENT CONSENT TO PARTICIPATE IN STUDY . . . 124 
CHAPTER 1

INTRODUCTION

Health care is in a state of transition and hospitals are merging to form integrated health care delivery systems in order to control costs, improve care, and remain competitive in a managed care marketplace (Dunn, 1996). The purpose of an integrated delivery system is to provide all types and levels of services required by a targeted population in a community (Fonner, 1996). Emergency care is one of these services. Many hospitals receive as high as $30 \%$ of their inpatient volume through the ED (Inguanzo \& Harju, 1985; Smeltzer \& Curtis, 1987). Providing quality care and satisfying emergency department patients can be considered an investment in the future growth of a health care organization through word of mouth, complaint behavior, and intention to return or recommend (Bendall \& Powers, 1995) .

Health care systems today are competing for covered lives. Hospital inpatient census has decreased dramatically due to the increase in managed care programs, decreases in elective surgeries with increases in uninsured Americans and a shift toward outpatient care to decrease costs. Administrators realize that patients have choices in 
determining which insurance plan to select and where to go for health care.

Patients today are more sophisticated and have higher expectations due to the role the media has played in educating the public and increasing their awareness of health care quality (Kanar, 1988). In response to these demands, health care organizations are focusing on the quality of care delivered. One way to measure the patients' perception of the quality of care is through patient satisfaction surveys.

First impressions are formed in the minds of patients as they arrive for emergency care. Satisfaction with the care received can carry over to an inpatient stay and may have an impact on patients' decisions to return in the future (Oliver, 1980). Satisfaction may then have a significant impact on a hospital's financial gain when paying or insured patients join Health Maintenance Organizations (HMOs) and request to return for hospitalization based on previous satisfying experiences with that organization.

Conversely, patient dissatisfaction can lead to negative feedback to referral sources. It can have a "multiplier effect" by discouraging future referrals. Satisfied consumers of products and services have been reported to praise a service to three other persons on average. By contrast, dissatisfied consumers share negative 
impressions with between 12 and 21 other people. Negative comments travel up to seven times faster than positive comments. Thus, the importance of minimizing dissatisfied consumers is imperative to help assure success in today's business world (Technical Assistance Research Programs Institute [TARP], 1986).

The actual purpose of emergency care is to evaluate, stabilize, and treat illnesses and injuries that need immeđiate attention as well as to provide treatment for conditions perceived by the patient as within this category. However, many of the poor and uninsured have been forced to obtain both emergency and basic health care in the ED due to lack of health care access elsewhere, resulting in severe overcrowding of emergency medical systems (Pane, Farner, \& Salness, 1991).

Nationwide, from 1985 to 1990, ED patient visits increased 19\%, from 84 million to 99.6 million, while total hospital admissions decreased by 7\%. Utilization studies have indicated that approximately $43 \%$ of the patients seen in emergency departments are considered non-urgent and only $17 \%$ are emergent (General Accounting Office [GAO], 1993). Emergent is defined as a classification of illness or injury that could be life- or limb-threatening and that nceds immediate attention. Urgent is defined as a classification of illness or injury that could be life- or limb-threatening if not treated within 2 to 6 hours. Non-urgent is an 
illness or injury that is neither limb- or life-threatening nor time sensitive. Many patients with minor illnesses use the ED because of lack of previous provider relationships, convenience, and the inability to make a prompt appointment with their private physicians (Shesser, Kirsch, Smith, \& Hirsch, 1991). The resultant problem is overuse and misuse of services resulting in prolonged waiting times for care which leads to dissatisfaction (Carey, Marshal1, Posavac, Talarowski, \& Abzug, 1983; Dershewitz \& Parchel, 1986; Inguanzo \& Harju, 1985).

The nurse has an opportunity to have an impact on the desired outcome of patient satisfaction. Specific behaviors may affect the patients' positive or negative opinions regarding their care. Because nurses are so highly visible in the delivery of health care, their role in contributing to patient satisfaction is critical (Bader, 1988). Research has demonstrated that satisfaction with nursing care is largely based on perception of the nurses' affective behavior toward the patient (Chang, Uman, Linn, Ware, \& Kane, 1984; Mangen \& Griffith, 1982; Oberst, 1984). The importance of nurses' behavior cannot be overlooked in addressing ways that nursing may have an impact on satisfaction with care.

Many patients who arrive in the ED are anxious due to the sudden event of injury or illness. Providing information to patients on $\mathrm{ED}$ arrival has been found to 
increase calmness and have a significant, positive effect on patients' perception of the quality of care and overall satisfaction (Bjorvell \& Steig, 1991; Krishel \& Baraff, 1993). Those who received information perceived the ability of the staff to decrease anxiety as significantly higher than those who did not receive the information.

Patients who present to the emergency department in hopes of immediate treatment may be greatly dissatisfied with prolonged delays. McMillan, Younger, and DeWine (1986) found that more than half of the patients who perceived a need to be immediately examined did not have this need met. They suggested that some type of communication to convince the patient that the staff understood and cared about the patient's condition be initiated.

The need for information from the nurse is apparent. Bjorvell and Steig (1991) found that patients who received the most information at the time of ED arrival were more satisfied with the general treatment, respect, and attitude later shown by staff than were the patients who had received no information at all on arrival. This lack of communication is what most frequently leads to patients' complaints (Gagnon, 1991).

A limited number of studies have focused on patient satisfaction in the ED environment. No studies were found that implemented nursing interventions to improve patient satisfaction and decrease anxiety in the ED setting. 
Because patient satisfaction with nursing care is a critical component in the provision of quality services in the $E D$, the topic is worthy of investigation. Anxious patients may choose the same health plan and return for future care to the same health care setting if their expectations are met or exceeded.

\section{Conceptual Framework}

The conceptual framework for this study was based on a model of service quality developed by marketing theorists (Parasuraman, Zeithaml, \& Berry, 1986). The primary objective for the supplier of any service should be consumer satisfaction. Within the health care arena the patient is the consumer of health care services and is equivalent to the consumer of market products within the service quality model. A health care organization may achieve its goals by satisfying the patient's goals. The supplier who recognizes and meets consumers' needs will increase the chance of having satisfied customers. Dissatisfaction will occur when a service fails to meet the customers' expectations.

The process for the formation of patients' expectations and how those expectations are met is summarized in Figure 1 (Tilbury \& Fisk, 1989). The left-hand column of this patient satisfaction model reflects the most common sources of patient expectations for health services: past experiences, comments from friends and doctors, and 


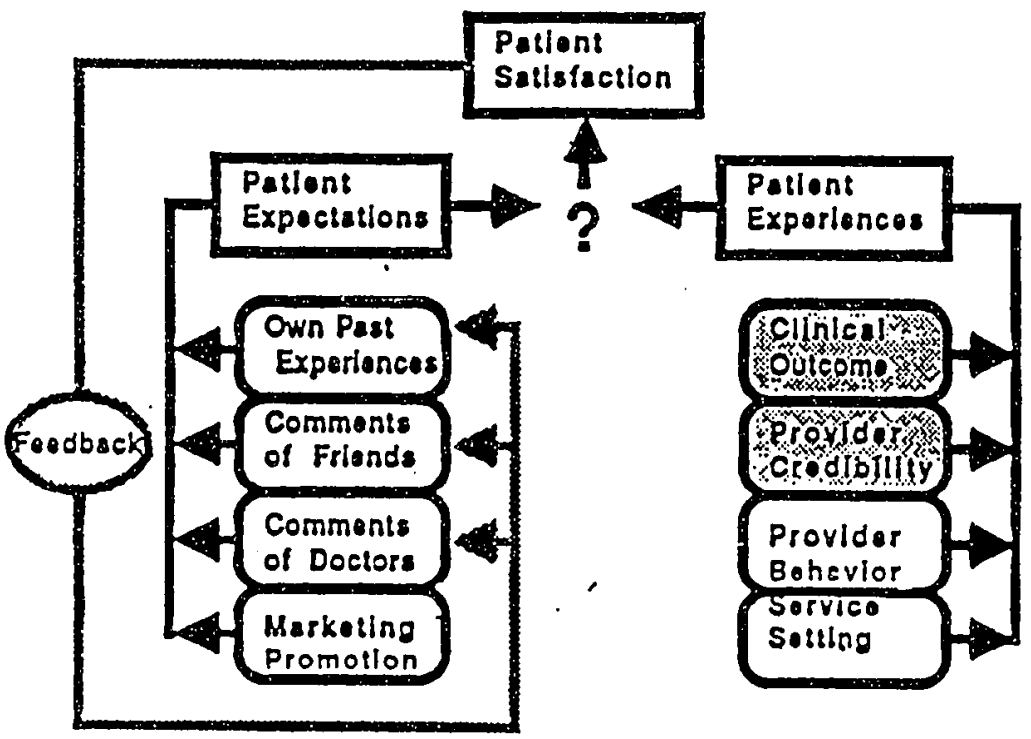

Figure 1. Patient satisfaction model (Tilbury \& Fisk, 1989). 
marketing promotion. Based on these components, a patient will arrive at the ED with certain expectations about the care they are about to receive.

The right-hand column shows the major components the consumer experiences during the encounter: clinical outcome, provider credibility, provider behavior, and service setting. During this process expectations are either confirmed or denied. The first component, the clinical outcome, is frequently considered a "given" by the patient and reflects the medical care the patient expects to receive. For example, a patient with a laceration assumes that he/she will be sutured by an ED physician who is trained and competent. The second component, provider credibility, is taken for granted by the consumer. Based on personal interviews with large numbers of recent users of various services, Parasuraman et al. (1986) found that most consumers attach a high credibility to the supplier that they have chosen. By the time of service, they believe in the supplier's competence. The supplier can destroy that belief by making an obvious error, such as misdiagnosing a patient's illness.

The third component, provider behavior, is considered the most crucial factor in meeting consumers' expectations (Parasuraman et al., 1986). In fact, many studies in health care have validated that nursing care is among the most important factors contributing to patient satisfaction 
(Tilbury \& Fisk, 1989). The best opportunity that nurses have to influence patient satisfaction is during the provision of those services. Thus, providing reassurance by the nurse, for example, would be expected to have a positive, significant effect on patient satisfaction. The intent is to strengthen the provider behavior to improve satisfaction and intent to return.

The final component, service setting, addresses the environment in which care is delivered. Examples would include the cleanliness of the ED, telephone availability, noise level, and privacy. The question mark on the model signifies the difference between the patient experience and the expectations which will determine satisfaction or possibly dissatisfaction with care received.

In summary, the patient arrives in the ED with preconceived expectations. The patient has predetermined that a successful clinical outcome will occur through care received by a credible provider. Although the service setting can significantly add to the patient's experience, it is the provider behavior which is the most important factor contributing to patient satisfaction. This patient satisfaction model supports the interventions used in this study by focusing on the provider behavior component. The nurse has the best opportunity to influence patient perceptions during the provision of patient care. Satisfaction or dissatisfaction is dependent on the various 
components described, but the behavior of the health care provider may be of significant importance.

\section{Purpose of the Study}

The purpose of this study was to investigate the effect of providing written information and reassurance on patient satisfaction, anxiety, and intent to return to the emergency department for future health care.

\section{Hypotheses}

The following null hypotheses were tested in the study:

1. There is ro significant difference in patient satisfaction, perception of anxiety, and intent to return for health care between patients who receive written information and those who do not.

2. There is no significant difference in patient satisfaction, perception of anxiety, and intent to return for health care between patients who receive reassurance and those who do not.

3. There is no significant difference in patient satisfaction, perception of anxiety, and intent to return for health care between patients who receive both written information and reassurance and those who do not.

4. There is no significant difference in patient satisfaction, perception of anxiety, and intent to return 
for health care between those who receive written information and those who receive reassurance.

5. There is no significant difference in patient satisfaction, perception of anxiety, and intent to return for health care between those who receive written information and those who receive both written information and reassurance.

6. There is no significant difference in patient satisfaction, perception of anxiety, and intent to return for health care between those who receive reassurance and those who receive both written information and reassurance.

\section{Definition of Terms}

For the purposes of this study, the following definitions of terms were used:

Patient Satisfaction (PS): The patient's judgment of the quality of care delivered (Donabedian, 1980). PS was measured using the Consumer Emergency Care Satisfaction Scale, CECSS (see Appendix A).

Anxiety: A condition characterized by subjective, consciously perceived feelings of tension and apprehension regarding current illness or injury (Clark, Fontaine, \& Simpson, 1994). Anxiety was measured by the Linear Analogue Anxiety Scale (LAAS) (see Appendix B).

Intent to Return: The likelihood that a person will return to the same ED if the need for emergency care occurs. 
Intent to return was measured by the Intent to Return Scale (Raper, 1994) (see Appendix C) .

The following interventions were used in this study:

Written Information: A written handout provided to the patient on ED arrival which explained the process for patient care (see Appendix D). Additional detail regarding the Written Information protocol will be provided in Chapter 3 .

Reassurance: A broad definition was used for the communication of reassurance by the researcher. Specific statements or actions initiated every 30 minutes by the researcher were categorized as: give information, apologize, ask a question, provide patient care, acknowledge patient presence, or reassure (see Appendix E). Additional detail regarding the Reassurance protocol will be provided in Chapter 3 .

\section{Summary}

The emphasis on patient satisfaction is consistent with the trend toward holding health care professionals accountable to the patient. Today's emergency departments are stressful, overcrowded environments. Anxious patients are requesting information and want to understand what is occurring within the ED environment. Nurses are challenged with addressing patient expectations for prompt and caring service. Providing written information and reassurance to 
address these needs may have a significant impact on the patients' perception of their experience in the ED, their anxiety level, and their intent to return for future health care. 
CHAPTER 2

REVIEW OF THE IITERATURE

The review of literature describes literature related to the study's dependent variables of patient satisfaction, anxiety, and intent to return. Satisfaction is further discussed in terms of consumers in general and, more specifically, patients receiving hospital care, emergency care, and nursing care. In addition, literature support for the study's interventions of providing written information and reassurance is presented.

\section{Satisfaction}

\section{Consumer Satisfaction}

Hospitals, as part of integrated health care delivery systems, are becoming more competitive and patients are now thought of as consumers and equal partners in the health care decision making process (Carter \& Mowad, 1988). A consumer is a person who buys and uses goods and services. Satisfaction is defined as a person's judgment of the quality of care delivered. Thus, a satisfied consumer of emergency services is one who perceives the quality of health care provided as positive. The importance of this concept for strategic survival of a health care organization 
is supported in the literature (Bailey, 1991; Elbeck, 1987). If patients are satisfied with care received at a given hospital, they are more likely to select their insurance plan and to return for future care resulting in larger numbers of health plan participants and financial income for the health care organization. In fact, service quality resulting in consumer satisfaction is one of the most emphasized terms in corporate life today. Zeithaml, Parasuraman, and Berry (1990) note that leading organizations are obsessed with service excellence. They use excellent service as their point of recognition to be different from others, to increase productivity, to earn the customers' loyalty, to encourage positive word-of-mouth advertising, and to seek some shelter from price competition. With service excellence, everybody wins.

To become competitive, a company must recognize that it doesn't sell products, but rather sells consumer satisfaction (Lele, 1988). The primary premise in business is that consumer satisfaction transforms into various forms of positive behavior, such as word-of-mouth communication, a belief that providers deliver excellence in health services, and tangible community support for health service funding (Churchill \& Supranant, 1982). These are general factors that make a positive impact on an organization.

Lele and Sheth (1988) identify four fundamentals of consumer satisfaction: the product, sales activity, after 
sales activity, and culture. Although this information is intended for the business industry, the concepts can be applied to the health care industry.

The first fundamental, product, includes such things as basic design, manufacturing, and quality control. In the health care industry the product reflects the quality of clinical nursing care provided in an efficient manner to the ill or injured. Staffing patterns, orientation classes, educational programs, and quality assurance all contribute to the final product. Quality emergency nursing care is then delivered by knowledgeable staff.

Sales activity, the second fundamental, is defined as messages the company sends out in advertising, how it chooses its sales force, and the attitudes they project to the customer. For example, promotional flyers, salespersons' attire, and courtesy of personnel all affect the consumers' impression of the company. The health care industry tries to project a feeling of caring and individualization as they advertise special services such as trauma, transplant, rehabilitation, and substance abuse treatment programs. The method used by staff as they care for patients is a sales activity as consumers' attitudes are altered or reinforced during the process of receiving care. Consumers evaluate services based on tangible evidence of caring such as a smile, tone of voice, eye contact, and successful problem-solving activities (Spicer, Craft, \& 
Ross, 1988). The nurses' awareness of salesmanship behaviors and the ability to communicate positively may influence patient satisfaction.

The third fundamental, after sales activities, includes warranties, parts, service, and complaint handling. Similarly, after health care has been provided, an organization can seek feedback from patients thr.רugh satisfaction surveys or telephone follow-up programs. Patient support groups or home health services may be established to demonstrate overall responsiveness to patients' concerns and needs. In effect, the patient may judge the hospital by its willingness to stand behind its product. Handled well, these activities help strengthen the patients' perception of the integrated health care delivery system they subscribe to.

Culture, the fourth fundamental of consumer satisfaction, reflects the values and beliefs of the firm and can be compared to a hospital's philosophy, mission statement, policies, and procedures. If the firm truly believes in the need for maximizing consumer satisfaction to ensure long-term success, then the product, sales activity, and after sales elements will coincide to deliver what the consumer expects and the organization wants. Similarly, the daily activities in a hospital will reflect the philosophy, mission statement, and policies working in conjunction to provide quality care and satisfied patients. 
Lele and Sheth's (1988) fundamentals can be used for diagnosing problems and developing a plan for improving consumer satisfaction in a wide range of industries. In the health care industry, and specifically in emergency care, the product, sales activity, after sales activity, and culture greatly influence patient satisfaction.

Today's patient is informed by the media about hospital and physician statistics and problems, by programs about health care, and by advertising that identifies characteristics of quality care such as timeliness of service, competence of practitioners, and statistics on the outcome of care. As integrated health care delivery systems emerge as the new health care delivery configuration, marketing efforts must focus on increasing the number of participants in the system health plan. Informed consumers enter a health care facility expecting greater professional attention, timely service, and uncomplicated treatment (Kanar, 1988). Even the less experienced consumer of health care will come to expect equal attention and service as a norm. An ED visit may be a person's first experience with the health care delivery system. This may have further implications if an ED visit results in hospitalization. Satisfaction with each phase of health care delivery is important to patients' perceptions of the integrated delivery system as a whole. 
When patients sense that their concern or apprehension is not being addressed by the staff, the end result may lead to broader perceptions of hospital inadequacies, frustrations, anger, and overall integrated delivery system concerns. These dissatisfied people may reconsider returning to a facility for future hospitalization and may select a different health plan--the one outcome an integrated delivery system hopes to prevent. Assessing patient satisfaction with hospital care becomes important from the integrated delivery system perspective, where the goal is to capture a population from birth through old age. Assuring satisfaction is key to this concept.

\section{Patient Satisfaction} with Hospital Care

The cognitive processes which lead a patient to feel satisfied are complex and dynamic. Many environmental factors contribute to patient satisfaction (PS). Previous patient satisfaction research has attempted to define and conceptualize the patient satisfaction process, develop reliable and valid instruments, and identify variables associated with PS. This research provides a basis for this study .

In conceptualizing the patient satisfaction process, a variety of definitions and dimensions of PS have been described in both the theoretical and empirical literature. As depicted in Table 1 there are at least three consistent 
Table 1

Dimensions of Patient Satisfaction by Author

\begin{tabular}{ll}
\hline Technical Dimension & \\
Technical-Professional & Risser (1978) \\
Technical Quality of Care & Ware, Davies-Avery, \& Steward (1978) \\
Technical Competence & Davis (1989) \\
Technical Care & Vuori (1987) \\
Medical-Technical & Chang, Uman, Linn, Ware, \& Lane (1984) \\
Technical Skils & Andrea (1991) \\
Medical Care & Heffring (1986) \\
Environmental Dimension & \\
Physical Environment & Ware, Davies-Avery, \& Steward (1978) \\
Waiting Lounge & McMillan, Younger, \& Dewine, (1986) \\
Amenities of Care & Vuori (1987) \\
Physical Environment & Andrea (1991) \\
Facilities & Heffring (1986) \\
Psychosocial Dimension & \\
Intra-personal & Risser (1978) \\
Trusting Relationship & Risser (1978) \\
Art of Care & Ware, Davies-Avery, \& Steward (1978) \\
Psychological Safety & Davis (1989) \\
Information-Giving & Davis (1989) \\
Psychosocial & Chang, Uman, Linn, Ware, \& Lane (1984) \\
Courtesy of Care & Chang, Uman, Linn, Ware, \& Lane (1984) \\
Psychosocial Skill & Andrea (1991) \\
Conmunication Skill & Andrea (1991) \\
\hline
\end{tabular}

Reproduced with permission of the copyright owner. Further reproduction prohibited without permission. 
patient satisfaction dimensions described by the majority of authors, although terminology may vary. These dimensions are technical, environmental, and psychosocial. The technical dimension addresses the medical treatment of the patient. All patients who present to the ED with a sudden illness or injury expect to receive competent medical care, i.e., laceration sutured or fracture casted. Quality care without complications is expected of the medical and nursing staff on duty. The patients' health care needs are generally met and the technical dimension of patient satisfaction is not an area of focus for this study. The environmental dimension or the physical surroundings may be a dissatisfier for patients and need improvement. This may or may not require capital expenditure. Minor changes in the environment can be initiated by nurses (i.e., color schemes, seating arrangements, magazine availability, access to refreshments, and cleanliness). However, nursing may have minimal control over some aspects of the environment due to the physical limitations of the structure or lack of available funds. The psychosocial dimension globally includes the communication between the patient and the health care professional. This dimension is frequently identified as one of the more important factors influencing PS with health care. Heffring (1986) surveyed 1,300 discharged patients regarding their satisfaction with hospital care. Results 
indicated that being treated as an individual and having timely, adequate information about the condition were more important to PS than the health care outcome. Pascoe and Attkisson (1983) determined that accessibility and convenience were significantly less important in influencing satisfaction than the personal dimension. The importance of the psychosocial component and the fact that nursing can possibly control and influence this dimension, provides a significant reason to pursue research on this dimension of patient satisfaction.

\section{Patient Satisfaction} with Emergency Care

In the emergency setting, the acuity of injuries and illnesses range from cold symptoms to cardiac arrest. Satisfaction has been shown to decrease as the need for emergency care becomes less urgent (Chande, Bhende, \& Davis, 1991; McMillan et al., 1986). Research related to PS with emergency care has focused on waiting times, information received, and acuity.

Patients presenting to the emergency department commonly wait extended lengths of time for treatment unless they are critically ill or injured. This wait may negatively influence their perceptions of care. DiGiacomo and Kramer (1982) studied factors causing ED delays with the goal of addressing these factors to enhance the quality of patient services. Forty percent of 420 subjects waited an 
average of 78 minutes. Positive, but insignificant, correlations were found between patient numbers, times of day, and waiting time. Therefore, investigators addressed other problems which became apparent during the study such as increasing the number of admitting personnel, altering staffing patterns, and adding patient care areas. No formal survey of patient satisfaction was conducted, but staff impressions indicated that patients were more positive about the care delivered after changes had been made to decrease waiting time. How this was assessed is not described.

Carey et al. (1983) used a patient survey as part of an investigation into the decrease in the number of emergency room admissions. Waiting time was suspected to be one of the critical issues. One hundred patients were interviewed regarding perceived quality of care, the quality of consumer relations, and waiting time. Statistically significant differences were found between patients who spent less than 2 hours in the emergency room compared to those who spent more than 2 hours. Of those who spent over 2 hours waiting, 15\% were dissatisfied with the information they received; only $4 \frac{\circ}{0}$ of those who were treated in less than 2 hours were dissatisfied $(\underline{p}<.05)$. When asked about their overall impressions of their emergency room experiences, no one who stayed under 2 hours had unfavorable impressions, while 14\% of those who stayed over 2 hours had unfavorable responses $(\underline{p}<.001)$. Waiting time affected the patients' reports of 
their initial impressions. Only $37 \%$ of those who stayed less than 2 hours had unfavorable initial impressions, compared to $24 \%$ of those with a longer waiting time ( $\underline{\mathrm{p}}<$ $.001)$. However, only 3\% said they would not return.

Concurring with previous study findings, Inguanzo and Harju (1985) found that the primary reasons given for patient dissatisfaction were delays in treatment and low levels of courtesy by staff. Patients noted that the staff either did not provide explanations for delays or they waited too long to give an explanation. This article did not describe the specific tool used for evaluation, the sample size, or the specific analytic methods, but results emphasized the need for frequent nurse/patient communication.

Bjorvell and Steig (1991) studied 187 patients who were discharged from the ED. They found that those who received the most information at the time of arrival at the ED were more satisfied with the general treatment $(\underline{p}<.05)$, respect $(\underline{\mathrm{p}}<.01)$, and attitude $(\underline{\mathrm{p}}<.05)$ later shown by the staff as well as with the information given later $(\underline{p}<.05)$ than were the patients who, on arrival, had received no information at all. Thus, the quality of the initial patient contact appeared to be of great importance in achieving patient satisfaction with care.

Patients with Iife-threatening injuries or illness receive priority in the $\mathrm{ED}$. Once their needs are met, 
minimizing waiting times for the remaining ED patients becomes the goal. Waiting perhaps cannot be avoided, especially for those not considered ill enough to require immediate treatment; however, it is clear from the literature that prolonged waiting times, as well as courtesy of staff and providing adequate information, has an impact on patient satisfaction.

The urgency of a situation is dependent on the individual's perception. Health care professionals identify the extent of a patient's illness based on pathophysiological knowledge and experience. However, lay persons may perceive their illness as very serious if it is a first time experience or simply based on lack of knowledge. A study of 10,253 ED patients examined prospectively patients' and physicians' perception of urgency of need for medical attention. Physicians' initial assessments indicated that $12.6 \%$ of patients needed attention immediately; 26.3\%, urgently; and $28.1 \%$, promptly. Patients' evaluations of urgency differed significantly (무 < .05) : 44.4\% thought they needed care immediately; $28.5 \%$, urgently; and 15.6\%, promptly (Gifford, Franaszek, \& Gibson, 1980).

Another study validated that significant differences existed between patients' and nurses' perceptions of the degree of illness urgency in the ED setting (Schultz, 1986). The results showed that the patient and the nurse saw the 
presenting condition as markedly different with respect to the degree of urgency (chi square $=40.54, \underline{\mathrm{n}}=64, \underline{\mathrm{p}}<.05$ ). of 64 patients interviewed, the majority were categorized as non-urgent by the nurse and only one third of patients' perceptions were congruent with the nurses'. Patients perceived themselves as requiring care more urgently than did the nurse.

The significance of the discrepancy in perception of the need for immediate care between the patient and the health care provider lies in how the nurse cares for and communicates with the patient. McMillan et al. (1986) investigated 368 ED patients' satisfaction with care and the results indicated that patients who perceived a need to be cared for immediately did not have this need met. If patients feel their needs are not being met appropriately or in a timely fashion, they are more likely to be dissatisfied with the care they receive. Higher levels of satisfaction are achieved when there is congruence between patient expectations and their actual experience (Bader, 1988). Thus, the nurse has the opportunity to provide information and reassurance at regular intervals and possibly establish realistic expectations for the patient. Research appears lacking in the area of initiating nursing interventions to influence $P S$ in the $E D$ setting. 
Patient Satisfaction

with Nursing Care

The importance of PS with nursing care has been described in the literature. Most PS research included nursing as only one of the services evaluated. In a study of services most crucial in forming patients' opinions about a facility, Carey and Posavac (1982) found that the perception of nursing care was the most crucial aspect in determining the overall satisfaction rating of the hospital. The 54-item instrument utilized was a standard survey developed for hospital use and included assessment of patient satisfaction with the hospital, nursing, housekeeping, food service and admitting. The process for identification of these dimensions is not reported nor are the psychometric properties of the instrument. This produces a limitation in the value of the content.

In a mail survey of 737 discharged patients, Lemke (1987) evaluated satisfaction with the following hospital services: nursing, housekeeping, admissions, food service, billing, lab, X-ray, respiratory therapists, escort personnel, social workers, and the chaplain. Medical care was omitted and psychometric properties of the Patient Satisfaction Survey were not reported. Results showed that when two patient groups were compared, those rating the hospital excellent and those rating it not excellent, the most significant difference was in their ratings of nursing service. However, psychometric limitations exist in terms 
of patients discriminating nursing from other hospital services.

Nursing staff are key to patient satisfaction. They are the hospital's frontline representatives. The nurse must balance the patients' needs for immediate, technical care while addressing the affective needs. Technical care is defined as a form of doing for the patient, a doing that includes skills, knowledge, physical care, and expertise in implementing health care. The affective dimension is a form of therapeutic relationship that includes kindness, information, verbal and nonverbal communication, and an opportunity for the patient to participate in the nursing process (Bader, 1988).

Because nurses are so highly visible in the delivery of health care, their impact in generating patient satisfaction is critical (Bader, 1988). Bader utilized the Patient Satisfaction Instrument developed by Hinshaw and Atwood (1982) to elicit information about satisfaction with specific nursing care behaviors. In identifying significant predictors of patient satisfaction, Bader found that $50 \%$ of nursing care behaviors are within the affective dimension of nursing, including sensitivity to people, listening, talking with patients, and demonstrating concern.

Wolf (1986) developed the Caring Behavior Inventory (CBI) by selecting from the literature words or phrases that represented caring. The highest ranked behaviors include 
both actions and attitudes that nurses considered important to the caring process in nursing. These included attentive Iistening, comforting, honesty, patience, responsibility and providing information. These same behaviors were identified by patients as important to their perception of care received. Thus, there is recognized agreement between patients and nurses regarding what nurses do to demonstrate caring.

Press and Ganey (1990) found that involving staff in interactions with family members and visitors contributed significantly to the patients' overall satisfaction. In addition, interpersonal issues exerted far more influence on patient satisfaction than experiences with technical aspects of care.

Mangen and Griffith (1982) and Oberst (1984) found that satisfaction with nursing care is largely based on patients' perceptions of the nurses' affective behavior. In contrast, several studies of patients who were considered acutely ill ranked technical behaviors as more important than the affective dimensions of nursing care (Larson, 1984; Robinson, 1978). This may occur in the ED setting when a patient presents with a life- threatening injury requiring immediate care.

Watson (1979) conducted a descriptive study to clarify caring behaviors and to identify similarities and differences between patients' and nurses' descriptions of 
caring. Caring can be perceived in terms of terhnical behaviors as well as affective behaviors of the nurse. Patients identified activities involving physical care and comfort as most indicative of caring such as medication and treatment procedures; whereas, nurses and nursing students identified more affective dimensions of care such as offering emotional support and listening.

Brown (1981) studied 80 hospitalized patients on medical-surgical units. Using a Likert-type scale, subjects rated the importance of nursing behaviors as indicators of care. Behaviors perceived as indicative of caring were a combination of what the nurse did (technical) and what the nurse was like as a person (affective). In addition, behaviors found to be fundamental to the perception of caring focused on physical well being, although the affective component of care was also important. The affective or psychosocial component of nursing care was a consistent, important theme in determining PS.

In summary, integrated health care delivery systems are competing for health plan participants. Patients' perceptions of an ED visit may influence future decisions to select a specific health plan. Nursing staff are highiy visible in the delivery of health care and are key to patient satisfaction. Prolonged waiting times, courtesy of staff, and providing adequate information have an impact on ED patient satisfaction. Nurses initiate both technical and 
psychosocial behaviors in the delivery of patient care. Both aspects have been found to be significant in determining patient satisfaction with nursing care. Implementation of a psychosocial intervention such as reassurance by the ED nurse is worthy of investigation.

\section{Anxiety}

Anxiety is a familiar experience. It is defined as a condition characterized by the patient's subjective, consciously perceived feelings of tension and apprehension regarding their current illness or injury. Patients may experience and express anxiety in many ways. Anxiety reactions can range from mild nervousness to panic and is a reflection of a number of underlying causes. It can reflect a normal response to stress, a pathological response, a physiological problem or a primary psychiatric disorder. For this study, the investigator was interested in situational anxiety related to the need for an ED visit.

$A$ visit to the $\mathrm{ED}$ is not commonplace for most individuals and the experience usually produces anxiety. Unfamiliar faces, a foreign environment, fear of the unknown, and prolonged waits are all factors which contribute to anxiety. One ethnographic study of an ED lobby described patients exhibiting anxious behaviors as they waited for care. For example, patients and family members repeatedly asked the ED nurse how much longer it 
would be before seeing a doctor. They made comments about how long they had been waiting. One person commented that they might die if they had to wait much longer. On several occasions, patients suggested to their family that they go elsewhere because they needed faster care. Many patients and family members would pace the lobby while they were waiting. All of these behaviors exhibited a certain level of anxiety at having to wait to be seen by a physician as perceived by the investigator. A limitation of this study was that patients were not interviewed to validate whether they were feeling anxious. It was possible that the investigator's perceptions were inaccurate (Andrea, 1990).

Emergency nurses must be aware of the psychological and psychosocial factors that are a part of the patient's visit to the ED. How the patient perceives the nurse may affect their levels of anxiety and the nurse may be able to make a difference in reducing anxiety.

\section{Intent to Return}

Behavioral intention is a concept primarily addressed in the marketing and business literature. Hospitals, as part of integrated health care delivery systems, like all enterprises, thrive only if they create satisfaction and loyalty in clients at an affordable cost. Fisk, Brown, Cannizzaro, and Naftal (1990) state that demand for an established organization, service, or product is generated 
by three mechanisms: repeat use by past clients, word-ofmouth recommendation from established users to new users, and attraction of new users by marketing communications.

The patients' intent to return is based on the assumption that satisfied patients will want to return to the same facility based on previous positive experiences. Hospitals, as part of integrated delivery systems, want to maintain market share in order to maintain census and minimize costs. In a competitive managed care environment, patients have a choice between selecting providers. Evaluating a patient's opinion regarding his/her intent to return provides further validation of service performance. Raper (1994) found that patient satisfaction with ED nursing care was a significant predictor of intent to return to the same $\operatorname{ED}(\underline{r}=.57, \underline{p}<.001)$. Using stepwise regression analysis, it was found that patient satisfaction with ED nursing care explained $28.6 \%$ of the variability of the intent to return to the ED. This is congruent with previous non-health care research (O'Connor, 1988; Oliver, 1980; Swan \& Trawick, 1981).

Swan, Sawyer, Van Matre, and McGee (1985) found that patient satisfaction was a much stronger predictor of intention to return to a specific hospital (gamma $=.705$, $\underline{t}=7.101)$ than directly measuring intention to revisit a hospital (gamma $=.196, \underline{t}=1.996)$. In addition, Woodside, Frey, and Daly (1989) studied patient satisfaction with 
received overnight hospital care utilizing multiple regression analysis. Telephone interviews were completed on 392 patients. They determined that patient satisfaction was effected by nursing care $\left(\underline{R}^{2}=.41, \underline{p}<.001\right)$ and directly related to intention to return to the hospital $\left(\underline{R}^{2}=.72\right.$, $\underline{p}<.001)$. Based on the results, training programs which focus on increasing the quality of information provided to patients by nurses were initiated.

Peyrot, Cooper, and Schnapf (1993) investigated outpatient satisfaction and intention to recommend outpatient health services $(\underline{N}=1,366)$. It seems reasonable to assume that if a patient would recommend a facility, they would also intend to return to the facility for future care. Two of the predictor variables of patient recommendation of the outpatient services included receiving enough prior information about the procedure $\left(\underline{R}^{2}=.29, \underline{p}<.05\right)$ and receiving enough information throughout the visit $\left(\underline{R}^{2}=.57\right.$, $\underline{p}<.05)$. Thus, intention to return to the hospital setting was influenced by the employee providing information to the patient, and established this as an important intervention in diagnostic services during an outpatient visit.

\section{Written Information Intervention}

Providing written information on ED arrival has been effective in increasing patient satisfaction. Krishel and Baraff (1993) studied 200 ED patients and found that 
patients who received information rated their overall satisfaction higher than did the control group ( $\mathrm{p}<.0001)$. Other items rated significantly higher were whether the patient would use the same ED again ( $\underline{p}<.0001)$, and the ability of staff to decrease anxiety $(\underline{p}<.0001)$. Although this intervention has been effective, only $11.8 \%$ of 93 California EDs were found to distribute written information to their patients (Krishel \& Baraff, 1993).

Anderson and Masur (1989) reported that patients who received information prior to procedures such as cardiac catheterization were less anxious than controls. This same concept can be applied to the ED setting whereby providing information about what will happen during an ED visit may help alleviate patients' fears and assist them to set realistic expectations regarding their ED visit.

\section{Reassurance Intervention}

Bursch, Beezy, and Shaw (1993) surveyed 258 ED patients to determine the importance of variables correlated with patient satisfaction with ED care. Two of the five most important variables included perceptions of nurse caring $\left(\underline{R}^{2}=.63, \underline{p}<.001\right)$ and the amount of information provided by nurses about what was happening with their care $\left(\underline{R}^{2}=\right.$ $.71, \underline{\mathrm{p}}<.001)$. This further validated the important role nurses play in providing information and reassurance to patients. 
Results of patient satisfaction surveys were utilized to address problems identified by patients at Thomas Jefferson University Hospital, Philadelphia. The concern was that unfavorable first impressions by patients admitted via the ED could be very detrimental to their overall satisfaction. In 1986, admitting patients from the ED was fairly easy due to a 75\% hospital occupancy rate. By late 1987, the growth in average occupancy had caused increased delays. Surveys indicated that satisfaction with the admission process was "somewhat" important to overall satisfaction and subsequent loyalty to the hospital. The actual length of the admission delay, however, was less critical than the degree of personal attention paid to the patients during the delay. Anticipating further increases in delays, administrators introduced a program of added attention to patients by ED personnel and patient representatives during peak hours. By 1989, delays increased and the perceived reasonableness of the delays diminished, but the perceived attention from staff during the delays increased sufficiently that overall satisfaction with the admission process did not deteriorate despite the longer delays (Fisk et al., 1990). Although specifics of the study design, population, interventions, and instruments used were not reported, the importance of providing information and reassurance to ED patients was validated. 
Badger (1994), in describing ways to allay anxiety, states that the nurse's physical presence and nonverbal behavior can greatly reduce a patient's anxiety. Appearing calm, interested, helpful, and offering appropriate reassurance is often the most effective treatment for a patient's anxiety. Much of a patient's anxiety may be based on exaggerated fears or lack of understanding about an illness. Patients presenting to the ED may be uncertain as to the seriousness of their illness. Lack of information can accelerate anxiety.

Peterson (1991) studied 72 patients undergoing cardiac catheterization. Half of the group received the typical information and education procedures, and the other half received social chit-chat by the nurse. Results showed that the social chit-chat was as effective as the educational intervention. This supported the power of the nurse presence and patient contact regardless of the content of what was discussed.

The primary intervention used to prevent severe anxiety levels is communication. Communication is sending, receiving, and understanding a message. It can be verbal or nonverbal. Sheehy (1992) describes four therapeutic communication techniques: supportive, silence, listening, and questions. The supportive technique can be useful in caring for an anxious patient. Examples include: verbalize support, acknowledge individual needs, and therapeutic 
touch. Silence as a nonverbal response can be a useful tool in therapeutic communication. Listening allows the nurse to hear the patient's concerns and is an active, physically visible process. Asking questions is part of the necessary data collection process. These techniques are used repeatedly by nurses providing patient care in all settings.

It has been theorized that there are two types of coping styles that are typically used by individuals faced with stressful events: they either seek out as much information as possible to make the event more predictable and thus more controllable or they avoid any information, preferring unpredictability (Miller \& Grant, 1979). This raises a question as to whether providing information is necessarily an intervention that will reduce anxiety. Research results have provided support for tailoring information to the patient's coping style to ensure maximum benefit (Watkins, Weaver, \& Odegaard, 1986). However, Peterson (1991) studied patient anxiety before cardiac catheterization and found that both educational and social intervention groups had a significant decrease in anxiety regardless of the patient's coping style when compared with the control group.

Recent research has been initiated to evaluate the effectiveness of a variety of modalities to reduce patient anxiety. Examples include utilizing: music for preoperative patients (Cirina, 1994), therapeutic touch and relaxation 
therapy in psychiatric patients (Gagne \& Toye, 1994), guided imagery in MRI patients (Thompson, 1994), bright light therapy in premenstrual patients (Cerda, 1994), and providing educational information in cardiac catheterization patients (Davis, Maguire, Haraphongse, \& Schaumberger, 1994). No research was found that utilized interventions which provided reassurance at regular intervals to reduce anxiety.

\section{Summary}

The literature related to consumer satisfaction, patient satisfaction, anxiety, and intent to return for emergency care was reviewed. Research related to the interventions of providing written information and reassurance was discussed. Nurses have the ability to influence patient satisfaction and reduce patient anxiety through the care they provide. Providing information, both in writing and through verbal communication, has been associated with higher levels of satisfaction, reduction in patient anxiety, and intention to return. Research that examined specific nursing interventions to address these variables in the ED setting were not found. As a result, providing written information and reassurance at regular intervals appear to be two possible approaches that could have an impact on patient satisfaction, anxiety, and intent to return to the $\mathrm{ED}$ for future care. A major objective of this study was to examine those interventions. 
CHAPTER 3

METHODOLOGY

This study was designed to determine if interventions consisting of written information, reassurance, or a combination of the two resulted in significant changes in patient satisfaction, anxiety, and intent to return to the ED for care. This chapter discusses the design, setting, sample, instrumentation, data collection procedures, pilot study, and data analysis for the study.

\section{Desion}

The design for this study was a posttest-only control group design involving a comparison between a control and three intervention groups (Campbell \& Stanley, 1963). Subjects were randomized to one of four groups. The control group received routine care consistent with existing ED practice standards. Intervention Group 1 received a written information sheet on ED arrival which explained what would occur during the ED visit (see Appendix D). Intervention Group 2 received reassurance from the nurse researcher at 30-minute intervals. This communication included specific information regarding their health care problem (i.e., lab results, specialist to arrive) or reassurance that they had 
not been forgotten (see Appendix E). Intervention Group 3 received a combination of written information and reassurance. The dependent variables were patient satisfaction, anxiety, and intent to return for care.

The use of a posttest-only design in this intervention study presents one major threat--the lack of a pretest to assure that the intervention and control groups were equal before the differential interventions occurred. Since a pretest would not have been appropriate in this study, randomization can suffice without a pretest to assure lack of initial biases between groups (Cook \& Campbel1, 1979).

\section{Setting}

The study was conducted in an emergency department in a community hospital located in a large city in southern California. The hospital, which is part of an extended integrated health care delivery system, has over 400 licensed beds and is a Level II trauma center. The ED has 23 beds and greater than 24,000 patient visits annually. Twenty percent of the patients arrive by ambulance and approximately $21 \%$ of the ED patients are admitted to the hospital for further treatment. The average length of ED stay is approximately $31 / 2$ hours. Forty-five percent of the patients arrive between 3:00 p.m. and 11:00 p.m. An average of 70 patients are seen per day. 
The nature of the community, nonprofit, hospital setting limits the generalizability of the study as there may be significant differences between private hospitals, public teaching hospitals, and community nonprofit hospitals in terms of the study variables which tend to be sensitive to the health care environment. In addition, it may not be possible to generalize the results to other geographic areas.

\section{Sample}

The target population consisted of adult, Englishspeaking patients who arrived in the ED and were classified within urgent or non-urgent categories by the triage registered nurse. Any patient in obvious distress, acute pain, or requiring immediate treatment was considered to belong in the emergent status category (i.e., difficulty breathing, chest pain, abdominal pain, etc.) and was excluded from the study. In addition, pediatric patients, non-English speaking, and patients with psychiatric complaints were excluded from the study population.

A total of 417 emergency department patients volunteered to participate in the study. Two hundred and forty (58\%) of these subjects completed and returned the questionnaires while 177 (42\%) did not. Out of the 240 subjects who participated in the study, 66 (28\%) completed 
the questionnaire while in the ED. The remaining 174 (72\%) returned them by mail.

Waltz, Strickland, and Lenz (1991) state it is not unusual to encounter response rates as low as $30 \%$ in questionnaire surveys. However, several techniques were used in this study which increased the response rate. These included personal contact with the subject in the ED, supplying a self-addressed and stamped return envelope, and follow-up telephone reminders.

A comparison of demographic variables for respondents and nonrespondents was completed to assess for response bias. Results showed no statistically significant differences between the groups based on age, gender, marital status, payor source, triage classification, first ED visit, disposition, or length of stay $(\underline{p}<.05)$. There was a statistically significant difference by race in subjects that did not return the questionnaires compared to those that did (chi square $=.00)$. A greater percentage of the Blacks $(20 \%, \underline{\underline{n}}=36)$ and Hispanics $(12 \%, \underline{\underline{n}}=21)$ were nonrespondents compared to those that did respond. There may have been a language barrier or lack of understanding for the Hispanic population.

Data were collected at varying times between $7 \mathrm{a} . \mathrm{m}$. and 11 p.m. between June 1, 1995, and November 14, 1995. Subject demographics, which were derived from the responses to the Demographic Questionnaire, are presented in Table 2. 
Table 2

Demographics of Subjects by Group

\begin{tabular}{|c|c|c|c|c|c|c|c|c|c|c|c|c|c|}
\hline & \multicolumn{2}{|c|}{$\begin{array}{c}\text { Group } 1 \\
(\text { Control) } \\
(\underline{\underline{n}}=63)\end{array}$} & \multicolumn{2}{|c|}{$\begin{array}{c}\text { Group } 2 \\
\text { (Written) } \\
(\underline{n}=57)\end{array}$} & \multicolumn{2}{|c|}{$\begin{array}{c}\text { Group } 3 \\
\text { (Reassure) } \\
(\underline{\underline{n}}=55)\end{array}$} & \multicolumn{2}{|c|}{$\begin{array}{c}\text { Group } 4 \\
\text { (Combo) } \\
(\underline{\underline{n}=65)}\end{array}$} & \multicolumn{2}{|c|}{$\begin{array}{c}\text { Total } \\
(\underline{N}=240)\end{array}$} & \multirow[b]{2}{*}{$x^{2}$} & \multirow[b]{2}{*}{$\underline{\mathrm{p}}$} & \multirow[b]{2}{*}{$\underline{\mathrm{H}}$} \\
\hline 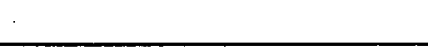 & $\underline{\mathrm{n}}$ & 8 & $\underline{\underline{n}}$ & 8 & $\underline{\mathrm{n}}$ & 8 & $\underline{\mathrm{n}}$ & 8 & $\underline{\underline{\mathrm{n}}}$ & $q$ & & & \\
\hline Age (in years) & \multicolumn{2}{|c|}{$\underline{M}=38$} & \multicolumn{2}{|c|}{$\underline{\underline{M}}=33$} & \multicolumn{2}{|c|}{$\underline{M}=41$} & \multicolumn{2}{|c|}{$\underline{\underline{M}}=33$} & \multicolumn{2}{|c|}{$M=36$} & & $.02 \star$ & 10.28 \\
\hline $\begin{array}{l}\text { Gender } \\
\text { Male } \\
\text { Female } \\
\end{array}$ & $\begin{array}{l}27 \\
36 \\
\end{array}$ & $\begin{array}{l}43 \\
57 \\
\end{array}$ & $\begin{array}{l}25 \\
32 \\
\end{array}$ & $\begin{array}{l}44 \\
56 \\
\end{array}$ & $\begin{array}{l}36 \\
19 \\
\end{array}$ & $\begin{array}{l}65 \\
35 \\
\end{array}$ & $\begin{array}{l}28 \\
37 \\
\end{array}$ & $\begin{array}{l}43 \\
57 \\
\end{array}$ & $\begin{array}{l}116 \\
124 \\
\end{array}$ & $\begin{array}{l}48 \\
52 \\
\end{array}$ & 8.39 & $.04 *$ & \\
\hline $\begin{array}{l}\text { Marital Status } \\
\text { Single } \\
\text { Married } \\
\text { Divorced } \\
\text { Widow } \\
\text { Separated } \\
\end{array}$ & $\begin{array}{r}25 \\
28 \\
6 \\
3 \\
1 \\
\end{array}$ & $\begin{array}{r}40 \\
44 \\
9 \\
5 \\
2 \\
\end{array}$ & $\begin{array}{r}33 \\
17 \\
3 \\
2 \\
2 \\
\end{array}$ & $\begin{array}{r}58 \\
30 \\
5 \\
3 \\
4 \\
\end{array}$ & $\begin{array}{r}25 \\
23 \\
2 \\
1 \\
4 \\
\end{array}$ & $\begin{array}{r}45 \\
42 \\
4 \\
2 \\
7 \\
\end{array}$ & $\begin{array}{r}31 \\
20 \\
8 \\
1 \\
5 \\
\end{array}$ & $\begin{array}{r}48 \\
31 \\
12 \\
1 \\
8 \\
\end{array}$ & $\begin{array}{r}114 \\
88 \\
19 \\
7 \\
12 \\
\end{array}$ & $\begin{array}{r}47 \\
37 \\
8 \\
3 \\
5 \\
\end{array}$ & 13.19 & .36 & \\
\hline $\begin{array}{l}\text { Race } \\
\text { White } \\
\text { Black } \\
\text { Hispanic } \\
\text { Asian } \\
\text { Other } \\
\end{array}$ & $\begin{array}{r}51 \\
6 \\
2 \\
3 \\
1 \\
\end{array}$ & $\begin{array}{r}81 \\
9 \\
3 \\
5 \\
2 \\
\end{array}$ & $\begin{array}{r}41 \\
8 \\
2 \\
3 \\
3 \\
\end{array}$ & $\begin{array}{r}72 \\
14 \\
4 \\
5 \\
5 \\
\end{array}$ & $\begin{array}{r}43 \\
4 \\
5 \\
1 \\
2 \\
\end{array}$ & $\begin{array}{r}78 \\
7 \\
9 \\
2 \\
4 \\
\end{array}$ & $\begin{array}{r}51 \\
7 \\
3 \\
2 \\
2 \\
\end{array}$ & $\begin{array}{r}78 \\
11 \\
5 \\
3 \\
3 \\
\end{array}$ & $\begin{array}{r}186 \\
25 \\
12 \\
9 \\
8 \\
\end{array}$ & $\begin{array}{r}78 \\
10 \\
5 \\
4 \\
3 \\
\end{array}$ & 6.55 & .89 & \\
\hline $\begin{array}{c}\text { Payor Source } \\
\text { Private } \\
\text { Medical } \\
\text { Self-Pay } \\
\text { CMS } \\
\text { Medicare } \\
\text { Other } \\
\end{array}$ & $\begin{array}{r}34 \\
10 \\
10 \\
6 \\
3 \\
0 \\
\end{array}$ & $\begin{array}{r}54 \\
16 \\
16 \\
9 \\
5\end{array}$ & $\begin{array}{r}25 \\
12 \\
11 \\
6 \\
3 \\
0 \\
\end{array}$ & $\begin{array}{r}44 \\
21 \\
19 \\
11 \\
5\end{array}$ & $\begin{array}{r}25 \\
11 \\
13 \\
1 \\
5\end{array}$ & $\begin{array}{r}45 \\
20 \\
24 \\
2 \\
9\end{array}$ & $\begin{array}{r}31 \\
15 \\
11 \\
4 \\
3 \\
1 \\
\end{array}$ & $\begin{array}{r}48 \\
23 \\
17 \\
6 \\
5 \\
1 \\
\end{array}$ & $\begin{array}{r}115 \\
48 \\
45 \\
17 \\
14 \\
1 \\
\end{array}$ & $\begin{array}{r}48 \\
20 \\
19 \\
7 \\
5 \\
1 \\
\end{array}$ & 10.5 & .79 & \\
\hline $\begin{array}{l}\text { Triage Classification } \\
\text { Nonurgent } \\
\text { Urgent }\end{array}$ & $\begin{array}{l}39 \\
24 \\
\end{array}$ & $\begin{array}{l}62 \\
38 \\
\end{array}$ & $\begin{array}{l}41 \\
16 \\
\end{array}$ & $\begin{array}{l}72 \\
28 \\
\end{array}$ & $\begin{array}{l}37 \\
18 \\
\end{array}$ & $\begin{array}{l}67 \\
33 \\
\end{array}$ & $\begin{array}{l}43 \\
22 \\
\end{array}$ & $\begin{array}{l}66 \\
34\end{array}$ & $\begin{array}{r}160 \\
80 \\
\end{array}$ & $\begin{array}{l}67 \\
33 \\
\end{array}$ & 1.4 & .71 & \\
\hline
\end{tabular}


Table 2--Continued

\begin{tabular}{|c|c|c|c|c|c|c|c|c|c|c|c|c|c|}
\hline & \multicolumn{2}{|c|}{$\begin{array}{c}\text { Group } 1 \\
\text { (Control) } \\
(\underline{n}=63)\end{array}$} & \multicolumn{2}{|c|}{$\begin{array}{c}\text { Group } 2 \\
\text { (Written) } \\
(\underline{\underline{n}}=57) \\
\end{array}$} & \multicolumn{2}{|c|}{$\begin{array}{c}\text { Group } 3 \\
\text { (Reassure) } \\
(\underline{\mathrm{n}}=55)\end{array}$} & \multicolumn{2}{|c|}{$\begin{array}{c}\text { Group } 4 \\
(\text { Combo) } \\
(\underline{n}=65) \\
\end{array}$} & \multicolumn{2}{|c|}{$\begin{array}{c}\text { Total } \\
(\underline{N}=240)\end{array}$} & \multirow[b]{2}{*}{$x^{2}$} & \multirow[b]{2}{*}{$\underline{p}$} & \multirow[b]{2}{*}{$\underline{\mathrm{H}}$} \\
\hline & $\underline{\mathrm{n}}$ & 8 & $\underline{\underline{n}}$ & 8 & $\underline{\underline{n}}$ & 8 & $\underline{\underline{n}}$ & 8 & $\underline{\mathrm{n}}$ & $q$ & & & \\
\hline $\begin{array}{l}\text { First ED Visit } \\
\text { Yes } \\
\text { No }\end{array}$ & $\begin{array}{l}10 \\
53 \\
\end{array}$ & $\begin{array}{l}16 \\
84 \\
\end{array}$ & $\begin{array}{l}11 \\
46 \\
\end{array}$ & $\begin{array}{l}19 \\
81 \\
\end{array}$ & $\begin{array}{l}13 \\
42 \\
\end{array}$ & $\begin{array}{l}24 \\
76 \\
\end{array}$ & $\begin{array}{l}13 \\
52\end{array}$ & $\begin{array}{l}20 \\
80 \\
\end{array}$ & $\begin{array}{r}47 \\
193 \\
\end{array}$ & $\begin{array}{l}20 \\
80\end{array}$ & 1.13 & .77 & \\
\hline $\begin{array}{c}\text { Disposition } \\
\text { Discharged } \\
\text { Admitted } \\
\end{array}$ & $\begin{array}{r}57 \\
6 \\
\end{array}$ & $\begin{array}{l}90 \\
10 \\
\end{array}$ & $\begin{array}{r}53 \\
4 \\
\end{array}$ & $\begin{array}{r}93 \\
7 \\
\end{array}$ & $\begin{array}{r}48 \\
7 \\
\end{array}$ & $\begin{array}{l}87 \\
13 \\
\end{array}$ & $\begin{array}{r}62 \\
3 \\
\end{array}$ & $\begin{array}{r}95 \\
5\end{array}$ & $\begin{array}{r}220 \\
20\end{array}$ & $\begin{array}{r}92 \\
8 \\
\end{array}$ & 2.8 & .42 & \\
\hline $\begin{array}{l}\text { Length of stay (in } \\
\text { minutes) }\end{array}$ & \multicolumn{2}{|c|}{$\underline{M}=185$} & \multicolumn{2}{|c|}{$\underline{M}=151$} & \multicolumn{2}{|c|}{$\underline{M}=146$} & \multicolumn{2}{|c|}{$\underline{M}=150$} & \multicolumn{2}{|c|}{$\underline{M}=158$} & & .06 & 7.3 \\
\hline
\end{tabular}

*Indicates statistically significant at .05 level. 
The average age of the subjects was 36 years with a range from 18 to 93 years. The average age was not equivalent across the four groups. The mean age for the Control group was 38 years, the Written Information group was 33 years, the Reassurance group was 41 years, and the Combination group was 33 years. The Kruskal-Wallis test showed that subjects in the four groups differed in age to a significant degree $(\underline{\mathrm{p}}<.05)$. The Written Information and the Combination groups were younger than the Control and Reassurance groups.

The entire sample was evenly distributed by gender, with $52 \%$ being female while 48\% were male. However, the chi-square results indicated a significant difference in gender across the groups (chi square $=8.38, \underline{p}=.04$ ). The Reassurance group had significantly more males and less females than the other three groups. This suggested a possible randomization failure and potential bias in the study.

The majority of subjects were either single (47\%) or married $(37 \%)$. The remainder were divorced (8\%), widowed (3\%), and separated (5\%). The majority of subjects, 186 (78\%), were white. The remaining subjects were Black (10\%), Hispanic (5\%), Asian (4\%), and Other (3\%). The largest proportion of the sample had private insurance $(\underline{\mathrm{n}}=115$, $48 \%$ ). This was followed by MediCal ( $\underline{n}=48,20 \%)$, Self Pay 
$(\underline{\underline{n}}=45,19 \%)$, County Medical Services (CMS) ( $\underline{\underline{n}}=17,7 \%$ ), Medicare $(\underline{\underline{n}}=14,5 \%)$, and $\operatorname{Other}(\underline{\mathrm{n}}=1,1 \%)$.

The majority of subjects were classified as non-urgent $(\underline{n}=160,67 \%)$ versus urgent $(\underline{\underline{n}}=80,33 \%)$. Eighty percent $(\underline{n}=193)$ of the patients had previously sought treatment in an emergency department. The majority of subjects were discharged home $(\underline{\underline{n}}=220,92 \%)$ and only $20(8 \%)$ were admitted to the hospital.

Chi-square results showed no significant differences between groups based on marital status ( $\mathrm{chi}$ square $=13.19$, $\underline{\underline{p}}=.36)$, race $(\mathrm{chi}$ square $=6.55, \underline{\mathrm{p}}=.89)$, payor $(\mathrm{chi}$ square $=10.45, \underline{p}=.79$ ), triage classification (chi square $=1.37, \underline{\mathrm{p}}=.71)$, first $\mathrm{ED}$ visit (chi square $=1.13, \underline{\mathrm{p}}=$ .76 ), or disposition (chi square $=2.81, \underline{p}=.42$ ).

Since the length of stay variable was significantly skewed, the Kruskal-Wallis test was used to determine significant differences between the four groups. Results showed no significant differences $(\underline{H}=7.31, \underline{p}=.06)$.

A post hoc power analysis to determine the adequacy of sample size at the .05 significance level was performed according to the procedures described by Cohen (1988). With a sample size of 59-60 subjects per each of the four groups and a medium effect size, the power for the Kruskal-Wallis analysis was .86. Munro, Visintainer, and Page (1986) recommend a power of at least .80 . It was concluded that 
the sample size was adequate to detect significant differences across groups.

\section{Instrumentation}

Three instruments were administered on a positest basis to both control and experimental groups. The Consumer Emergency Care Satisfaction Scale (CECSS) instrument was utilized to evaluate patient satisfaction with ED nursing care and the modified Linear Analogue Anxiety Scale (LAAS) was used to evaluate patient anxiety. Five questions comprised the Intent to Return scale as developed by Raper (1994). The investigator received permission to use copyrighted instruments prior to data collection.

Consumer Emergency Care

Satisfaction Scale (CECSS)

The CECSS (see Appendix A) was developed and tested in a series of three studies (Davis, Bush, \& Thomas, 1996). In the first study, Davis (1989) used concept analysis, Risser's Patient Satisfaction Scale (1975), and personal experience in emergency nursing care to generate a 25-item instrument. The items were then critiqued for content validity by a panel of five content specialists. Percentage of agreement ranged from .75 to 1.00 for scale items with an overall agreement of .91. One item that produced an agreement of .60 was deleted and additional items proposed by the specialists were addea, resulting in a 29-item scale. 
In study 2, persons completing the CECSS responded to each item on a 5-point Likert-type scale ranging from completely agree (5) to completely disagree (1). There were 25 items in the psycholgoical safety subscale and 4 items in the physical safety subscale. Ten of the items were negatively worded. Scoring for negatively worded items in the CECSS was reversed. A higher score indicated a higher level of satisfaction.

Persons from four southern communities in Texas and Kentucky participated to assess the psychometric properties of the CECSS. The availability sample consisted of persons who had recently experienced emergency nursing care ( $\underline{n}=97$ ) and patients currently in the emergency department ( $\underline{n}=$ 137).

Construct validity of the CECSS was tested using principal components factor analysis. The criterion set for significant factor loading was .40 , with .30 being the minimally acceptable value (Burns \& Grove, 1993). Revisions ot the subscales were necessary due to the results of the factor analysis. Four factors were retained and subscales renamed as Psychological Safety, Discharge Teaching, Information Giving, and Technical Competence. The final instrument consisted of 20 items.

The instrument was evaluated for internal consistency using Cronbach's alpha coefficient. The CECSS demonstrated a Cronbach's alpha coefficient of .92 , with the 
Psychological Safety, Discharge Teaching, Information Giving, and Technical Competence subscales demonstrating alpha coefficients of $.92, .93, .67$, and .75 respectively. The objective of study 3 was to confirm the factor matrix using Multiple Groups Factor Analysis. Aggregate data $(\underline{N}=468)$ from four researchers and clinicians were used to confirm the subscale matrix. As a result of the factor analysis, one item was deleted due to incongruence with other clustered items. Two items dealing with the technical competence of the nurse were included with other items to form a factor dealing with caring. This created three hypothesized factors: Caring--12 items, Teaching--3 items, Dissatisfaction--4 items, which were again subjected to the Multiple Groups Factor Analysis.

As in the original factor analysis done in study 2 , the negatively worded items were clustered together. In contrast with study 2, however, the negatively worded items did not load significantly on any other factor. The decision was made to use the negatively worded items as filler items to minimize response set and exclude them from scoring since the existence of a "dissatisfaction" subscale did not seem appropriate.

Finally, Cronbach's alpha coefficient was again used to test the internal consistency of items in the subscales. The Caring subscale demonstrated an alpha of 0.92 , while the Teaching subscale was found to have an alpha of .87 . 
Testing of the CECSS is continuing. Nurses in at least 10 states have used or are using the instrument (Davis \& Bush, 1995). Davis and Bush (1995) have concluded that the CECSS has demonstrated adequate psychometric properties suggesting it is ready for further testing of satisfaction with emergency nursing care.

The 19-item scale, with a potential range of scores between 15 and 75, was used in this study. The scoring procedures for the CECSS are included in Appendix A. Since the subscales of Caring and Teaching coincide with the study interventions of providing reassurance and information, total scale scores were used for comparisons. The internal consistency reliability coefficient for the CECSS scale for this study was .96 , indicating high reliability of the instrument.

\section{Anxiety}

Linear Analogue (Visual Analogue) Scales have been used to measure subjective phenomena (such as pain and anxiety) in a variety of clinical settings. Reviews have concluded that these scales are simple, sensitive, and reproducible instruments (Gift, 1989; Huskisson, 1983; Wewers \& Lowe, 1990). Visual analogue scores tend to correlate positively with scores on 10-point verbal scales (Carlsson, 1983). An important characteristic and potential limitation of visual analogue scales is that they measure only one dimension of a phenomenon; for example, its strength or intensity (Waltz et 
al., 1991). Visual analogue scales' major advantages are their ease of use, acceptance by respondents, and sensitivity to subtle fluctuations in levels of the stimulus. Minimal time and effort demands on ill respondents make the visual analogue scale useful in the emergency department setting.

In this study, the Modified Linear Analogue Scale (LAAS) was used to measure patient anxiety. The LAAS is a $10-\mathrm{cm}$ line with extremes of total calm and extreme anxiety (see Appendix B). Subjects were asked to mark the number that reflected their level of anxiety. Responses ranged from $0-10$.

\section{Intent to Return}

Intent to return to the emergency department for future care was determined by a 5-item Likert-type adaptation of a bipolar adjective scale (Raper, 1994). Scoring yields a summated score with a potential range from 5 to 25 . A high score indicates a strong likelihood that subjects intend to return to the same ED for future emergency needs. This instrument was used in a study of 200 ED patients which examined patient satisfaction with ED nursing care, patient acuity, and intention to return to the ED (Raper, 1994). Cronbach's coefficient alpha was .87 for the Intent to Return Scale. The mean score was 20.7 with a standard deviation of 5.527. The distribution was negatively skewed 
and leptokurtotic (Raper, 1994). Validity measurements of the instrument are not describeà.

In this study, the Intent to Return Scale yielded an alpha coefficient of .89, which indicated a high internal consistency for the instrument. The distribution of scores in this study were negatively skewed and leptokurtotic, similar to the findings described by Raper (1994).

\section{Demographic Data}

Demographic data were collected to describe the patient sample (see Appendix F). Item content focused on age, gender, marital status, triage class, length of stay, number of previous ED visits, race, disposition, and payor source.

\section{Data Collection}

Human subjects approval for the research study was obtained from the University of San Diego's Committee for the Protection of Human Subjects (see Appendix G) and the hospital Institutional Review Board (see Appendix H).

\section{Pilot Study}

Due to the high degree of environmental variability in the study setting, a pilot study was conducted to evaluate the data collection procedure. In 19 hours on two consecutive days, 19 patients agreed to participate in the study. Despite their initial willingness to complete the study questionnaire upon arrival to the ED, none of the 19 
subjects were actually willing to complete the study questionnaire on discharge. Subjects were then given the option to complete the questionnaires at home and return them by mail. They were provided with a self-addressed stamped envelope. All 19 subjects in the pilot study agreed to do so, although only 11 (58\%) actually returned the surveys .

Minor changes were made in the data collection process to assist in tracking subjects and their assigned study groups. For example, bed number and chief complaint were added to the demographic data sheet. Paperwork for each subject was clipped together for ease of administration. Questionnaire packets were color coded according to assigned group to assist the researchers in the data collection and intervention process. Finally, self-addressed stamped envelopes were added to each packet to have available for the subjects who refused to complete the survey in the ED.

It was determined during the pilot study that one research assistant would be necessary to assist in data collection. The researcher and assistant collected data together for a period of 12 hours following a demonstration, discussion, and return demonstration process to assure consistency in the data collection procedure. A script, which described what to say when approaching the patient to explain the study and seek consent for participation, was written to further increase control in the process. The 
subject consent form was revised to account for the research assistant.

\section{Data Collection Process}

Patients who arrived in the emergency department during data collection times between June 1 and November 14, 1995, were approached to participate in the study. On arrival to the ED, the ED staff nurse brought the patient into the private triage area for evaluation. The RN asked the patient routine assessment questions which included name, birth date, chief complaint, medical history, private physician, and allergies. At that point, the ED nurse assigned a triage classification of emergent, urgent, or non-urgent. Emergent patients were brought directly into the ED for immediate care and these patients were not considered eligible for the study due to the severity of their illness. In addition, some urgent patients had significant discomfort (i.e., pain, nausea, and dizziness) and were excluded from study participation. For remaining patients that were stable, the researcher or the research assistant identified herself. The nature of the study and potential risks and benefits were described to the patient. Following consent to participate (see Appendix I), the subject was then asked if they had previously been a patient in an ED and to rate their current level of anxiety on the LAAS. Precautions were undertaken so that no patient care delay occurred during the research study. For example, if 
the patient was called to move to registration during the researcher and subject interaction, the researcher stopped the data collection process. Once the subject was again waiting, data collection was resumed.

Subjects were told that they had the right to withdraw at any time without consequences to the health care they were receiving. They were told that all data would remain anonymous and only group data would be analyzed. In addition, it was stated that there were no wrong answers and that their opinions, whether positive or negative, were valued. All subjects received the usual care provided by the ED staff nurses.

\section{Study Intervention Protocols}

Following completion of the LAAS, subjects were randomized to one of four groups. This technique is the all-purpose procedure for achieving pretreatment equality of groups, within known statistical limits (Cook \& Campbell, 1979). For example, the first subject was assigned to the Protocol 1 (Control) group, the second subject to the Protocol 2 (Written Information) group, the third subject to the Protocol 3 (Reassurance) group, and the fourth subject to the Protocol 4 (Combination) group. This process of consecutive assignment was repeated as patients consented to participate in the study. This process was occasionally difficult to achieve in the ED setting. For example, 
eligible subjects were randomized to one of four groups. A subject may have been randomized to the group which required reading the written information sheet. However, some patients stated they couldn't read anything due to their clinical condition (i.e., severe headache, photophobia, nausea, vomiting, or abdominal pain). At that point they were assigned the next group which did not require reading the information sheet. The next subject was assigned to the written information group. This clinical necessity introduced a potential internal validity threat by decreasing the effectiveness of the random assignment technique. However, it occurred only rarely.

\section{Protocol 1 (Control)}

Subjects assigned to the control group did not receive treatment interventions beyond those routinely provided during ED visits. At the time of discharge, the ED staff nurse notified the researcher that care for the subject had been completed. The subject was asked to again rate his/her perception of anxiety, and to complete the CECSS and the Intent to Return questionnaire. If the subject preferred to complete the questionnaires at home, they were provided with a self-addressed stamped envelope with which to return questionnaires by mail within 1 week. It would have been ideal if all of the subjects were willing to complete the questionnaires prior to leaving the ED. However, after prolonged waiting times, subjects were very anxious to 
leave. The option was to risk refusal to participate, which seemed highly likely based on the pilot study, or allow the mailing option in hopes of minimizing biased data due to the lack of a representative ED sample. If the questionnaires were not returned within 1 week, a follow-up reminder telephone call was placed.

\section{Protocol 2 (Written Information)}

The first experimental intervention consisted of providing the subject with written information regarding what would occur during the emergency department visit. It was given to the subject upon arrival in the ED, and it took less than 5 minutes for the subject to read. The 2-page summary (see Appendix D) provided the subject with general information about the process that patients experience in the ED. It also provided some of the reasons why waiting might be occasionally necessary. The subject was asked to read the written information and the researcher answered any questions regarding it. At the time of discharge, the data collection process was completed as described above for Protocol 1 .

\section{Protocol 3 (Reassurance)}

The second experimental intervention consisted of providing the subject with reassurance at 30 -minute intervals. The nurse researcher provided reassurance by making contact with the subject and stating, if the subject 
was waiting in the lobby, that they would be brought in as soon as possible. If the subject was waiting in the ED, the nurse researcher provided information regarding his/her current status (i.e., waiting for physician evaluation, lab or $\mathrm{X}$-ray results pending, prescriptions to be written, etc.). This communication was meant to reassure the waiting subject that they had not been forgotten. The research nurse documented each time reassurance was provided to the subject and coded this information on the Reassurance Data Collection Form (see Appendix E) to assure the 30-minute interval time frames were met.

In addition, each reassurance intervention was coded according to the following reassurance categories: give information, apologize, ask questions, provide patient care, acknowledge patient presence, and reassure. Examples of specific statements or actions for each category are described in Appendix E. To strengthen the control in the intervention process, comparisons between the total number and types of reassurance given for the two groups that received the Reassurance intervention were completed. Kruskal-Wallis tests showed that there were no significant differences between the Reassurance and Combination groups for the total number of reassurance contacts made nor the type of reassurance intervention provided (see Table 3 ). This validated the consistency of the reassurance intervention initiated by the researchers across groups. 
Table 3

Kruskal-Wallis Test for Reassurance Intervention Differences by Group

\begin{tabular}{|c|c|c|c|c|c|c|}
\hline $\begin{array}{l}\text { Reassurance } \\
\text { Intervention }\end{array}$ & $\begin{array}{l}\text { Mean } \\
\text { Rank }\end{array}$ & Cases & Group & $\underline{\mathrm{H}}$ & $\underline{d f}$ & $\underline{p}$ \\
\hline $\begin{array}{l}\text { Total Number of } \\
\text { Contacts }\end{array}$ & $\begin{array}{l}60 \cdot 47 \\
60 \cdot 52\end{array}$ & $\begin{array}{l}55 \\
65\end{array}$ & $\begin{array}{l}\text { Reassurance } \\
\text { Combination }\end{array}$ & .0001 & 1 & .99 \\
\hline Give Information & $\begin{array}{l}58 \cdot 41 \\
62.27\end{array}$ & $\begin{array}{l}55 \\
65\end{array}$ & $\begin{array}{l}\text { Reassurance } \\
\text { Combination }\end{array}$ & .398 & 1 & .53 \\
\hline Apologize & $\begin{array}{l}58 \cdot 13 \\
62 \cdot 51\end{array}$ & $\begin{array}{l}55 \\
65\end{array}$ & $\begin{array}{l}\text { Reassurance } \\
\text { Combination }\end{array}$ & .685 & 1 & .41 \\
\hline Fsk Questions & $\begin{array}{l}59 \cdot 70 \\
61.18\end{array}$ & $\begin{array}{l}55 \\
65\end{array}$ & $\begin{array}{l}\text { Reassurance } \\
\text { Combination }\end{array}$ & .055 & 1 & .81 \\
\hline $\begin{array}{l}\text { Provide Patient } \\
\text { Care }\end{array}$ & $\begin{array}{l}62.11 \\
59.14\end{array}$ & $\begin{array}{l}55 \\
65\end{array}$ & $\begin{array}{l}\text { Reassurance } \\
\text { Combination }\end{array}$ & .356 & 1 & .55 \\
\hline $\begin{array}{l}\text { Acknowledge } \\
\text { Patient Presence }\end{array}$ & $\begin{array}{l}60.61 \\
60.41\end{array}$ & $\begin{array}{l}55 \\
65\end{array}$ & $\begin{array}{l}\text { Reassurance } \\
\text { Combination }\end{array}$ & .002 & 1 & .97 \\
\hline Reassure & $\begin{array}{l}56.43 \\
63.95\end{array}$ & $\begin{array}{l}55 \\
65\end{array}$ & $\begin{array}{l}\text { Reassurance } \\
\text { Combination }\end{array}$ & 1.93 & 1 & .16 \\
\hline
\end{tabular}


The 30-minute interval was met without difficulty except on 9 out of 594 (1.5\%) occasions. In five instances, the subjects were in the radiology department for procedures such as computerized tomography, scans, ultrasounds, and intravenous pyelograms, and were inaccessible to the researcher. On three occasions, the subjects were sleeping and a decision was made to not awaken them. On one occasion, the ED physician was discussing a serious situation with the subject and contact could not be provided for a 40-minute interval. These instances were so rare that this posed a minor limitation.

Finally, patients received the reassurance intervention a variable number of times since it was dependent on how long they had to wait for emergency care. For example, a patient who was in the ED for 2 hours received reassurance four times, whereas a patient waiting 5 hours received the reassurance 10 times. All patients were included regardless of waiting time since waiting time will always be variable based on the ED census and patient acuity. Length of stay was calculated for potential comparisons to determine if the number of reassurance intervention episodes represented a control issue for the study. The average length of stay for the group receiving the reassurance intervention was 146 minutes and the average length of stay for the group receiving written information and reassurance was 150 minutes. At the time of discharge, the data collection 
process was completed as previously described for Protocol 1 .

Protocol 4 (Combination)

The third experimental intervention was a combination of Protocols 2 and 3 . In essence, subjects were asked to read the information sheet on ED arrival and the researcher provided verbal reassurance at 30-minute intervals. At the time of discharge, the data collection process was completed as previously described for protocol 1.

\section{Data Analysis Techniques}

Data were analyzed through a process of data reduction, hypotheses testing and post hoc analysis. The statistical Package for the Social Sciences (SPSS-PC) was used for computer data analysis. Descriptive analysis of the demographic data was completed. Frequencies, percentages, means, standard deviations, and scattergrams were performed, as well as tests of skewness and kurtosis to determine normal distributions of data.

Significance testing for skewness and kurtosis on all subjects was completed (see Table 4). Results indicated that both Anxiety-pre and Anxiety-post scores were positively skewed and the CECSS and Intent to Return scores were negatively skewed to a significant degree ( $\underline{0}<.05$ ). In addition, Anxiety-pre scores were platokurtotic, whereas Anxiety-post, CECSS, and Intent to Return scores were 
Table 4

Test for Variable Skewness and Kurtosis

\begin{tabular}{l|c|c|c|c|c}
\hline Variable & Skewness & $\begin{array}{l}\text { S.E. } \\
\text { Skew }\end{array}$ & Kurtosis & $\begin{array}{c}\text { S.E. } \\
\text { Kurt }\end{array}$ & $\underline{\text { N }}$ \\
\hline $\begin{array}{l}\text { Anxiety- } \\
\text { Pre }\end{array}$ & $.50 *$ & .16 & $-.84 *$ & .31 & 240 \\
$\begin{array}{l}\text { Anxiety- } \\
\text { Post }\end{array}$ & $1.94 *$ & .16 & $4.41 *$ & .31 & 240 \\
CECSS & $-1.33 *$ & .16 & $1.18 *$ & .31 & 239 \\
Intent & $-1.29 *$ & .16 & $1.15^{*}$ & .31 & 236 \\
\hline
\end{tabular}

${ }^{\star} \underline{p}<.05$. 
leptokurtotic $(\underline{p}<.05)$. Therefore, the non-normality was significant. Due to the severe skewness of the data, the Kruskal-Wallis test, a nonparametric test for ordinal data, was utilized. Since data could be ordered by rank, the assumption of normality and homogeneity of variance were eliminated. The only assumption about the form of the scores is that they are at least ordinal in scale (Jarrell, 1994). The Kruskal-Wallis test is analogous to the ANOVA for two or more groups.

The Kruskal-Wallis test for significant differences between groups was used to analyze the data. Pairwise differences were analyzed to determine if the groups differed from one another. In addition, Spearman correlation coefficients were determined to assess the relationship between age (which was not equally distributed) and the dependent variables of satisfaction, anxiety, and intent to return scores.

\section{Summary}

The methodology used in this study was the posttestonly design involving a control and three intervention groups. The setting and a pilot study were discussed. Specific sample characteristics were delineated including differences noted between the four groups. Instrumentation and the data collection process were outlined, inclusive of 
the four intervention protocols. Finally, the data analysis techniques were described. 


\section{CHAPTER 4}

\section{RESULTS AND DISCUSSION}

The primary thrust of the research was to investigate the effect of providing written information and reassurance on patient satisfaction, anxiety, and intent to return to the ED for future care.

\section{Data Analysis Related to the Research Hypotheses}

Six null hypotheses were tested using the KruskalWallis test. Results are depicted in Tables 5 through 8 . A significance level of .05 was set for testing the hypotheses. None of the null hypotheses were refuted as stated. Thus, there were no statistically significant differences among the groups.

Hypothesis 1 stated that there would be no significant difference in patient satisfaction, perception of anxiety, and intent to return for health care between patients who received written information and those who did not.

Hypothesis 2 stated that there would be no significant difference in patient satisfaction, perception of anxiety, and intent to return for health care between patients who received reassurance and those who did not. 
Table 5

Kruskal-Wallis Test for Anxiety-Pre by Group $(\underline{N}=240)$

\begin{tabular}{c|c|c}
\hline Mean rank & Cases & Group \\
\hline 105.82 & 63 & 1 = Control \\
117.79 & 57 & $2=$ Written Information \\
122.79 & 55 & $3=$ Reassurance \\
135.17 & 65 & $4=$ Combination \\
\hline
\end{tabular}

$\underline{\mathrm{H}}=5.98 ; \underline{\mathrm{df}}=3 ; \underline{\mathrm{p}}=.1126$. 
Table 6

Kruskal-Wallis Test for Anxiety-Post by Group ( $\underline{N}=240)$

\begin{tabular}{c|c|c}
\hline Mean rank & Cases & Group \\
\hline 108.07 & 63 & 1 = Control \\
125.25 & 57 & 2 = Written Information \\
124.95 & 55 & $3=$ Reassurance \\
124.62 & 65 & $4=$ Combination \\
\hline
\end{tabular}

$\underline{H}=3.0738 ; \underline{d f}=3 ; \underline{p}=.3804$. 
Table 7

Kruskal-Wallis Test for CECSS by Group ( $\underline{N}=239)$

\begin{tabular}{c|c|c}
\hline Mean rank & Cases & Group \\
\hline 101.64 & 62 & 1 = Control \\
121.19 & 57 & $2=$ Written Information \\
127.45 & 55 & $3=$ Reassurance \\
130.16 & 65 & $4=$ Combination \\
\hline
\end{tabular}

$\underline{H}=6.6688 ; \underline{\mathrm{df}}=3 ; \underline{\mathrm{p}}=.0832$. 
Table 8

Kruskal-Wallis Test for Intent to Return by Group (N $=236$ )

\begin{tabular}{c|c|c}
\hline Mean rank & Cases & Group \\
\hline 109.33 & 62 & 1 = Control \\
112.94 & 55 & $2=$ Written Information \\
128.18 & 54 & $3=$ Reassurance \\
123.92 & 65 & $4=$ Combination \\
\hline
\end{tabular}

$\underline{\mathrm{H}}=3.2281 ; \underline{\mathrm{df}}=3 ; \underline{\mathrm{p}}=.3578$. 
Hypothesis 3 stated that there would be no significant difference in patient satisfaction, perception of anxiety, and intent to return for health care between patients who received both written information and reassurance and those who did not.

Hypothesis 4 stated that there would be no significant difference in patient satisfaction, perception of anxiety, and intent to return for health care between patients who received written information and those who received reassurance.

Hypothesis 5 stated that there would be no significant difference in patient satisfaction, perception of anxiety, and intent to return for health care between patients who received written information and those who received both written information and reassurance.

Hypothesis 6 stated that there would be no significant difference in patient satisfaction, perception of anxiety, and intent to return for health care between patients who received reassurance and those who received both written information and reassurance.

\section{Discussion}

The discussion of the results addresses randomization, dependent variables, instrumentation, and intervention issues. Since all of these issues cross over the 
hypotheses, they will be addressed categorically instead of by individual hypothesis.

\section{Randomization}

Since there was a difference in gender by group, there was the concern of bias in the study. Therefore, it was necessary to determine if there was gender difference based on the dependent variables. Kruskal-Wallis tests showed that there was a significant gender difference for both the Anxiety-Pre score and the CECSS (see Table 9). The CECSS scores were significantly higher for males than females ( $\underline{p}<$ .05). Gender difference did not significantly affect the Anxiety-Post scores nor the Intent to Return scores which suggests no bias. Therefore, the failure to randomize successfully by gender is of greatest concern when looking at the Anxiety-Pre scores and the CECSS scores.

The other variable that was not equally distributed across groups was age. Since age is considered interval data, Spearman correlation coefficients were determined to see if age was correlated with the dependent variables (see Table 10). Results showed a positive, significant correlation between age and CECSS, indicating that older subjects had higher scores on the CECSS. Age compared with Anxiety-Pre, Anxiety-Post, and Intent to Return scores was not statistically significant. 
Table 9

Kruskal-Wallis Test for Gender Differences

\begin{tabular}{l|c|c|l|c|c|c}
\hline \multicolumn{1}{c|}{ Variable } & Mean rank & Cases & Sex & H & $\underline{\text { df }}$ & $\underline{\underline{\mathrm{D}}}$ \\
\hline Anxiety-Pre & 111.46 & 116 & Male & 3.883 & 1 & $.0488^{* *}$ \\
& 128.96 & 124 & Female & & & \\
Anxiety-Post & 112.38 & 116 & Male & 3.4457 & 1 & .0634 \\
CECSS & 128.10 & 124 & Female & & & $.0207 * *$ \\
& 130.47 & 116 & Male & 5.3530 & 1 & \\
Intent to Return & 110.13 & 123 & Female & & & .6521 \\
\hline
\end{tabular}

${ }^{*} \mathrm{p}=.05$. 
Table 10

Spearman Correlation Coefficients for Age and Dependent Variables

\begin{tabular}{|c|c|c|c|}
\hline Variable & Correlation & Cases & $\underline{p}$ \\
\hline Anxiety-Pre & -.0360 & 240 & .579 \\
\hline Anxiety-Post & -.0319 & 240 & .623 \\
\hline CECSS & .2110 & 239 & $.001 * *$ \\
\hline Intent to Return & .0870 & 236 & .183 \\
\hline
\end{tabular}

$* * \underline{p}<.05$. 
Therefore, the failure of randomization due to age and gender was only significant with respect to the CECSS. The older and the male subjects tended to have higher scores.

Examination of the means for CECSS by group indicated that the gender bias in the randomization was such that the Reassurance group was mostly males. The Reassurance group and the Control group were also older. Since both males and older subjects scored higher on the CECSS, the bias that was introduced by the randomization failure resulted in higher CECSS scores in the Reassurance group than if the randomization had been successful. This means that the mean CECSS score in the Reassurance group was higher than it should have been. However, the Control group mean was actually lower than the mean score in the Reassurance group. So even if the Reassurance group mean was a few points lower, it would still be equal to the control group mean. Furthermore, there was no significant difference between the CECSS scores across the groups. Therefore, it was concluded that the small amount of bias introduced by age and gender randomization failure was not enough to alter the results or conclusions drawn.

\section{Patient Satisfaction}

Patient satisfaction scores were high for all groups. Ninety percent $(\underline{N}=215)$ of the subjects were satisfied according to CECSS scoring procedures (Davis \& Bush, 1995). 
This finding of high satisfaction scores is consistent with other research on ED patients (Raper, 1994).

Even though satisfaction scores were high, evaluating patient satisfaction with nursing care in the emergency department setting was difficult due to the multiple variables affecting satisfaction. For example, it was difficult for subjects to separate their feelings about the nurse when perhaps they were dissatisfied with the physician care or the extended wait for care. Some subjects crossed off the word "nurse" on the CECSS and wrote in "Doctor." The measurement tool did not account for physician issues. Depending on the subject's needs, the ED nurse may have spent minimal time with the subject. For example, some subjects only spoke with the ED nurse to provide basic information. The health care assistant may have taken the vital signs and the subject was seen and discharged by their private MD or the ED physician. When it was time for the subject to fill out the 19-item questionnaire, it may have been difficult for the subject to accurately evaluate the nursing care due to minimal interaction with the nurse.

One other control problem was related to the number of nurses who cared for the subject during the ED visit. Although subjects were instructed to evaluate the nurse they had the most contact with, it was confusing to the subjects to determine one nurse to evaluate. This issue was evident based on comments written on the forms. Subjects wrote in 
the margins addressing which nurse they evaluated on the CECSS. For example, "The first nurse I spoke with was rude and in a hurry. However, the other two nurses were great!" Since scores were high, it appeared the subject rated the second two nurses.

Although nurses are expected to introduce themselves to patients, it did not always occur. There are many team members who participate in patient care in the ED setting used for this study. For example, respiratory therapists initiate the breathing treatments, EKG technicians complete the electrocardiograms, X-ray technicians complete the $\mathrm{X}$ rays, phlebotomists draw the blood samples, and health care assistants transport patients and do vital signs. Since all team members wear scrubs, it is difficult for patients to differentiate the nurses from the other team members. At the end of the ED Visit, it may have been difficult for the subject to solely evaluate the nursing care. It may have been more appropriate to evaluate all team members individually, rather than trying to evaluate nursing only. Given the complexity of the environment, it was the researcher's goal to identify ways that nursing could influence the patients' perception of the ED experience. It may have been difficult for the patients to evaluate nursing only when they may have had other concerns unrelated to the nursing care. 
Anxiety

There were several issues encountered with respect to the concept of anxiety and how it was measured in the study. These issues may have contributed to the nonsignificant results.

One issue of concern was the apparent variation in subjects' understanding of the term anxiety. Comments made by subjects when documenting their level of anxiety included, "I'm always anxious," "I'm always stressed," "my job is stressful," and "my anxiety has nothing to do with the ER." This led the researcher to understand that levels of anxiety may not be related to the ED issues at all and, therefore, the interventions being tested may have had no effect on the subjects' anxiety. Some subjects referred to anxiety as a personal trait, rather than considering their level of anxiety as related to the specific situation and $E D$ visit.

In addition, there was a lack of understanding of the term anxiety. A few subjects asked the researcher what exactly was meant by anxious. This occurred during the pilot study.

There were 12 subjects who documented a higher level of anxiety on discharge from the ED in comparison to when they arrived. This in essence was the opposite of what was anticipated to occur. In discussing this with subjects at the time, various factors came into play. For example, one 
subject was diagnosed with a kidney stone that would require lithotripsy treatment. This occurred on a Friday night and the lithotripsy procedure could not be scheduled until Monday morning. He was very concerned about having to get through the weekend with the unbearable discomfort. He was relieved that the source of the pain was identified and treatable, but was anxious about the delay in definitive treatment. He, therefore, rated his anxiety higher on discharge for that reason.

Another subject was diagnosed with a sexually transmitted disease. The implication of infidelity by her significant other was of great concern to the subject. She was very upset at time of discharge and the increase in her anxiety level reflected that.

One subject stated that her increase in anxiety at discharge had nothing to do with the care received in the ED but chose not to elaborate further. Another subject's anxiety went from a 1 to a 5 on discharge due to the fact that her insurance company had denied authorization for payment of the ED visit. Regardless, she stayed for treatment. She was angry with her insurance company and concerned about the bill she would have to pay. Thus, her increase in anxiety.

One subject stated her anxiety level changed from a 5 to a 10 on discharge because she felt she was treated rudely 
by the ED physician and ignored by the ED staff. This subject was in the control group.

One subject rated his anxiety from a 1 to a 6 because his ankle turned out to be fractured. This meant a longer recovery period and a cast requirement. The implications of being unable to work in his current job were of concern to him.

Three subjects rated their anxiety levels higher on ED discharge because their injury required surgery which they were not expecting. Two subjects stated they were upset with the long wait and one subject had a higher anxiety level on discharge due to a disappointing diagnosis.

These clinical situations were difficult to control and anticipate. A single score on an anxiety instrument does not take into consideration clinical situations that subjects may be facing. It may not have been possible to alter the subjects' anxiety level with the interventions used.

It is interesting to note that 52 of the 240 subjects (22\%) rated their anxiety level as 0 on both arrival and discharge from the ED. It is difficult to know if the subjects were unwilling to admit that they were anxious based on some underlying need to be stoic and calm or whether there was measurement error with terminology issues. The researcher frequently noted discrepancies in observed behavior and facial expressions in comparison to their 
rating of "not anxious." Questions such as "Do you think I will be o.k?" "How long will it be til I see a doctor?" "I'm really sick," "I need something now for pain" all seemed to reflect some level of anxiety. However, they rated their anxiety level as 0 . This may reflect measurement error or a sensitivity issue given the ED environment.

It is possible that the higher acuity patients that were not included in the study had higher anxiety levels. Perhaps the interventions would have been significant for that population.

\section{Intent to Return}

Intent to Return scores were also high. Eighty-three percent of the subjects had scores greater than 15 (possible range was 5 to 25). One hundred subjects (42\%) scored 25 out of 25 on this variable. The mean score was 20.80 with a standard deviation of $5.13(\underline{N}=236)$. This is consistent with previous results found by Raper (1994) $(\underline{\mathrm{M}}=20.7$, $\underline{\mathrm{SD}}=$ 5.5, $\underline{\mathrm{N}}=200)$. In that study, patient satisfaction with $\mathrm{ED}$ nursing care significantly contributed to the patient's intention to return to the $\mathrm{ED}$.

The high scores may simply be related to the hospital's geographic location from the subject's home. In an emergency, patients may go to the closest facility regardless of previous experience with the facility. 
Several subjects did not respond to the questions and wrote in the margins that they did not live in the area.

Since many patients have the option to select their health benefits annually, and the goal is to recruit more health plan participants, a better question may have been, "Would you select this health plan in the future?"

\section{Written Information Intervention}

The lack of significant differences between the groups which received written information and those that did not is inconsistent with previous research results (Krishel \& Baraff, 1993). A possible explanation for the lack of significant difference includes the weakness of the intervention. Although providing adequate information on $E D$ arrival has been related to improved satisfaction (Bjorvell \& Steig, 1991), the actual information on the sheet provided may not have met the subjects' needs. Since $80 \%$ of the subjects had previously sought care in an ED, they may have known what to expect, been less anxious, and been more familiar with the process and environment. Desensitization to the environment may have weakened the intervention effect for the study variables. For those subjects who stated they had been to an ED previously, it is unknown how many times they had sought ED care. It is possible that the intervention may have been more effective for first time ED patients. 
There wasn't a clear linkage between the written information sheet provided and the dependent variables. Perhaps a more specific question related to the subjects' perception of the value of the information sheet would have been useful.

\section{Reassurance Intervention}

In this design, the nurse researcher initiated the intervention while the ED staff nurses provided the usual care. This was done to allow the usual care to take place. However, it was then difficult to ascertain whether the subjects included the researcher's intervention of reassurance when evaluating their satisfaction using the CECSS.

Because satisfaction and intent to return scores were so high, it may have been impossible to initiate any intervention to significantly increase the scores. It is possible that the staff nurses were providing such excellent care as their routine care that the interventions had little effect and were minimally different from what was already occurring. Had the usual care been withheld, an ethical limitation would have been created and difficult to justify.

Conversely, there may be other interventions than the ones used in this study that would prove to be effective in the ED setting. In addition, the instruments utilized to evaluate the dependent variables may have lacked the sensitivity to differentiate between the groups. For 
example, if anxiety had been measured utilizing an instrument that clearly defined the concept for the subject, results may have been different due to a better understanding of the concept. Perhaps questions related specifically to the effectiveness of the interventions used would have resulted in significant differences between the groups. The CECSS may not have evaluated the interventions specifically.

\section{Summary}

Chapter 4 included the results of the study. Data analyses related to the research hypotheses were described. The discussion addressed the issues of randomization, patient satisfaction, anxiety, and intent to return. The lack of significant differences among groups was also discussed in relationship to the interventions utilized. In addition, control and measurement limitations were described. 
CHAPTER 5

SUMMARY AND IMPLICATIONS

This chapter includes a summary of the research Iindings, the conceptual framework, and implications for nursing research, clinical practice, administrative practice, and education.

\section{$\underline{\text { Summary }}$}

This study evaluated the effect of providing written information and reassurance on patient satisfaction, anxiety, and intent to return to the ED for future care. Four hundred and seventeen patients volunteered to participate in the study and 240 (58\%) patients returned completed questionnaires.

The subjects were randomized into one of four groups: control, written information, reassurance, and combination of written information and reassurance. All subjects were asked to rate their level of anxiety on arrival and discharge from the ED, and to complete the CECSS and the Intent to Return scale. Demographic data were collected and described.

Data were analyzed with the SPSS-PC program. Due to the lack of a normal distribution for all three dependent 
variables, the nonparametric, Kruskal-Wallis test was used to compare group differences. Results demonstrated no significant difference between any of the groups $(\underline{D}<.05)$. In addition, a post hoc power analysis supported the adequacy of the sample size.

The majority of subjects rated their anxiety as low; their satisfaction as high, and their intent to return as high.

\section{Conceptual Framework}

The conceptual framework for this study was based on a model of service quality which addresses consumer expectations and the consumer experience as driving satisfaction. The patient satisfaction model in Figure 1 specifies the various components which can lead to patient satisfaction. The interventions utilized in this study were aimed at enhancing the provider behavior to significantly effect patient satisfaction. Although there were no significant differences between the four groups, the model fit as a depiction of the process. Areas for improvement include strengthening the sensitivity of the instrument used to measure satisfaction. The ED experience may require the use of a broader instrument which measures other aspects of emergency care besides the nursing component. In addition, the original conceptual model of service quality developed by Parasuraman et al. (1986) may be of beneficial use when 
used in conjunction with the Service Quality (SERVQUAL) instrument. The SERVQUAL instrument encompasses five service-quality dimensions: tangibles, reliability, responsiveness, assurance, and empathy. This would allow the patient to evaluate more than just the nursing care. Rather than measuring the outcome of patient satisfaction, the SERVQUAL instrument is used to evaluate the gaps between consumer expectations and perceived satisfaction with the service. By evaluating the service-quality gaps, one could assess the effect of specific interventions on key dimensions of emergency service.

\section{Implications}

\section{Nursing Research}

The results from the current investigation suggested that future research address definitional issues, instrumentation, sampling, and design. It is recommended that the definition of anxiety be clarified for future research studies. Further research needs to be done in evaluating patients' level of anxiety. Differentiation between anxiety as a personality trait versus situational anxiety may be necessary to assure that the correct variable is understood and measured. Refinement of instruments to accurately measure anxiety and satisfaction is indicated. Perhaps focusing interventions on patients who identify high anxiety levels would be more effective. A qualitative study 
focusing on patients' perception of anxiety and satisfaction in the ED setting and recommendations for nursing interventions would help identify future directions for nursing research and care.

Conducting intervention research in the emergency care setting posed numerous challenges for the researcher. Difficulty in achieving randomization due to clinical patient conditions would need to be addressed in future studies. Since there was a significant difference by race between nonparticipants and participants, further research in this area is indicated.

It would be worthwhile to repeat this study and train a group of ED nurses to provide the intervention during their usual care. This would eliminate some of the confusion over which nurse to evaluate when completing the CECSS. It is also suggested to compare first time ED users with repeat users since their needs and expectations may be significantly different. The acute status patients were not included in this study nor were non-English speaking patients. Further research inclusive of these populations would be worthwhile since results would be more representative of the ED patient population.

Further research in this area is imperative in order to strategically plan interventions that will most efficiently improve patient satisfaction, minimize patient anxiety, and 
ensure their intent to return to the same facility for future care.

\section{Clinical Practice}

Implications for clinical practice address interventions and nursing behaviors that should be incorporated into the ED setting. Although providing written information to patients on ED arrival did not result in significant differences in this study, it may be a worthwhile and inexpensive mechanism to inform patients about what will occur during their visit. It may be of more benefit to first time ED users. In addition, ED nurses should consider assessing patients' level of anxiety routinely on arrival. This will help identify if a problem exists that can be resolved. An increased awareness will assist nurses in providing adequate information, reassurance, or other interventions as appropriate.

Nurses need to assess, recognize, and manage patient expectations through communication. Identification of specific expectations of nursing care sets the stage for mutual goals. In addition, unrealistic expectations of nursing care can be explored and negotiated. If the nurse shares with the patient that lab results will not be completed for 2 hours, the patient will not expect the results in 1 hour. Improved satisfaction and less anxiety may be the end result. 


\section{Administrative Practice}

The results of this investigation have implications for administrative practice. Satisfaction with health care is an important outcome associated with new and return business. Leadership commitment to make patient satisfaction a priority is essential. Developing and utilizing accurate, repetitive, and useful instruments to measure patient satisfaction is the basic building block of any effective system.

Instituting formal guest relations programs for employees may prove beneficial in establishing norms of behavior and seek changes in personnel behavior, as needed. Albert (1989) describes the development of service-oriented culture as necessary to meet consumer expectations. If specific nursing behaviors are identified which contribute to improved satisfaction and less anxiety, classes should be offered which reinforce those interventions into daily practice. Nurse administrators need to be actively involved in the effort to ensure a service-oriented culture.

\section{Nursing Education}

The results of this investigation also have implications for nursing education in academia as well as in the hospital setting. Nursing curricula must incorporate patient satisfaction as a desired outcome of nursing care (Davis-Martin, 1986). Attitudes, behaviors, and interventions that improve satisfaction and minimize anxiety 
should be identified, discussed, and demonstrated to beginning nursing students as well as experienced nurses. Critical thinking exercises which focus on situations resulting in patient dissatisfaction, various solutions to promote satisfaction, and professional behaviors incongruent with patients' needs and desires should be discussed and implemented. Simulations via interactive video may be worthwhile to strengthen communication skills. Staff education could focus on the patient as a consumer and ways to communicate with anxious as well as dissatisfied patients. Collaboration between academia and service is essential to incorporate the concept of patient satisfaction into mainstream nursing education (Greeneich, 1993).

\section{Conclusions}

This investigation evaluated the effect of providing written information and reassurance on patient satisfaction, anxiety, and intent to return for future care. The results showed no significant differences between any of the groups, meaning the interventions did not have a significant effect on the dependent variables.

However, patient satisfaction scores were high, anxiety scores were low, and patients intended to return for future care. Other interventions may have been able to alter the scores to a significant degree. Research to identify potential interventions is needed. 
Further research is necessary to strengtinen instrumentation, resolve methodological issues, and identify ways that the ED nurse can improve care provided to all ED patients which will result in minimal anxiety, improved satisfaction, and intent to return in the future.

The ED setting is a complex environment with multiple factors affecting patients' perceptions of the experience. In addition, the concepts of anxiety and satisfaction in this setting are also complex and difficult to measure with current instruments. Nursing care is but one facet of the entire picture, making it difficult to isolate from the other aspects of emergency care such as medical treatment, waiting time, access, cost, and environment. Continued attempts need to be made to conduct clinical research in the ED related to satisfaction and anxiety if improvements are to be achieved in the nursing care provided to the emergency department patients. 


\section{REFERENCES}

Albert, M. (1989). Developing a service-oriented health culture. Hospital Health Care Administration, 34(2), $167-83$

Anderson, K. O., \& Masur, F. T. (1989). Psychologic preparation for catheterization. Heart and Lung, 18, $154-163$

Andrea, J. (1990). Ethnographic study of an ED lobby. Unpublished manuscript.

Andrea, J. (1991). Development of an instrument to measure patient satisfaction with emergency care. Unpublished manuscript.

Bader, M. M. (1988). Nursing care behaviors that predict patient satisfaction. Journal of Nursing Quality Assurance, 2 $(3), 11-17$.

Badger, J. M. (1994, May). Calming the anxious patient. American Journal of Nursing, pp. 46-50.

Bailey, M. M. (1991). What the future holds for ED nursing. Nursing, 91, 56-59.

Benda11, D., \& Powers, T. L. (1995). Cultivating loyal patients. Journal of Health Care Marketing, 15 (4), 5053. 
Bjorvell, H., \& Steig, J. (1991). Patients' perceptions of the health care received in an emergency department. Annals of Emergency Medicine, 20 (7), 734-738.

Brown, L. (1981). Behaviors of nurses perceived by hospitalized patients as indicators of care. Dissertation Abstracts International, 40 (4361-B). (University Microfilms)

Burns, N., \& Grove, S. K. (1993). The principles of nursing research: Conduct, critique, and utilization (2nd ed.). Philadelphia: Saunders.

Bursch, B., Beezy, J., \& Shaw, R. (1993). Emergency department satisfaction: What matters most? Annals of Emergency Medicine, $\underline{22}(3), 586-591$.

Campbel1, D. T., \& Stanley, J. C. (1963). Experimental and quasi-experimental designs for research. Chicago: Rand McNally College Publishing Company.

Carey, R. G., Marshall, G., Posavac, E., Talarowski, E., \& Abzug, H. (1983). Waiting time and patient satisfaction with emergency room care. Emergency Health Services Quarterly, 1(4), 37-40.

Carey, R. G., \& Posavac, E. J. (1982). Using patient information to identify areas for service improvement. Health Care Management Review, I(2), 43-48.

Carlsson, A. (1983). Assessment of chronic pain I: Aspects of the reliability and validity of the visual analogue scale. Pain, $\underline{6}(6), 87-101$. 
Carter, S., \& Mowad, L. (1988). Is nursing ready for consumerism? Nursing Administration Quarterly, $12(3)$, 74-78.

Cerda, G. M. (1994). The effects of bright light therapy on symptoms of depression, anxiety, and hibernation in patients with premenstrual syndrome. Journal of Women's Health, $\underline{3}(1), 5-15$.

Chande, V., Bhende, M., \& Davis, H. (1991). Pediatric emergency department complaints: A three-year analysis of sources and trends. Arnals of Emergency Medicine, $\underline{20}(9), 1014-1016$.

Chang, B., Uman, G., Linn, L., Ware, J., \& Lane, R. (1984). The effect of systematically varying components of nursing care on satisfaction in elderly women. Western Journal of Nursing Research, $\underline{6}, 367-379$.

Churchill, G. A., \& Supranant, C. F. (1982). An investigation into the determinants of customer satisfaction. Journal of Marketing Research, 19, 491504.

Cirina, C. L. (1994). Effects of sedative music on patient preoperative anxiety. Today's OR Nurse, 16(3), 15-18. Clark, S., Fontaine, D., \& Simpson, T. (1994, August Suppl.). Recognition, assessment, and treatment of anxiety in the critical care setting. Critical care Nurse, pp. 2-6. 
Cohen, J. (1988). Statistical power analysis for the behavioral sciences. New York: Academic Press. Cook, T. D., \& Campbell, D. T. (1979). Quasiexperimentation: Design and analysis issues in field settings. Chicago: Rand McNally.

Davis, B. A. (1989). Consumer satisfaction with emergency department nursing care: Instrument development (Doctoral dissertation, Texas Women's University, 1988). Dissertation Abstracts International, 49 , 2433A.

Davis, B. A., \& Bush, H. A. (1995). Developing effective measurement tools: A case study of the Consumer Emergency Care Satisfaction Scale. Journal of Nursing Care ouality, $\underline{9}(2), 26-35$.

Davis, B. A., Bush, H. A., \& Thomas, S. W. (1996). Measuring consumer satisfaction with emergency department nursing care. Manuscript submitted for publication.

Davis, T. M., Maguire, T. D., Haraphongse, M. , \& Schaumberger, M. (1994). Preparing adult patients for cardiac catheterization: Information, treatment and coping style interactions. Heart and Lung, 23(2), 130139.

Davis-Martin, S. (1986). Outcome and accountability: Getting into the consumer dimension. Nursing Management, $17(10), 25-27$. 
Dershewitz, R., \& Parchel, W. (1986). Patients who leave a pediatric emergency department without treatment. Annals of Emergency Medicine, 15, 717-720.

Digiacomo, E. V., \& Kramer, L. D. (1982). A study of emergency unit waiting time. Quality Review Bulletin, $\underline{8}(11), 10-13$.

Donabedian, A. (1980). Explorations in quality assessment and monitoring (Vol. 1). Ann Arbor: Health Administration Press.

Dunn, D. (1996). Health care 1999: A national bellwether. Journal of Health Care Finance, $\underline{22}(3), 23-27$.

Elbeck, M. (1987). An approach to client satisfaction measurement as an attribute of health service quality. Health Care Management Review, 12(3), 47-52.

Fisk, T. A., Brown, C. J., Cannizzaro, K., \& Naftal, B. (1990). Creating patient satisfaction and loyalty. Journal of Health Care Marketing, $10(2), 5-15$. Fonner, E. (1996). Identifying comets on the road to integration. Journal of Health Care Finance, 22 (3), $28-33$

Gagne, D., \& Toye, R. C. (1994). The effects of therapeutic touch and relaxation therapy in reducing anxiety. Archives of Psychiatric Nursing, $\underline{8}(3), 184-$ 189 . 
Gagnon, L. (1991). Customer service: Is the answer better communication? Journal of Emergency Nursing, 17(2), $63-64$.

General Accounting office. (1993). Growth and change in emergency department use (Report No. GAO/HRD 93-4). Washington, DC: Government Printing Office.

Gifford, M. J., Franaszek, J. B., \& Gibson, G. (1980). Emergency physicians' and patients' assessments: Urgency of need for medical care. Annals of Emergency Medicine, $\underline{9}(10), 502-507$.

Gift, A. G. (1989). Visual analogue scales: Measurement of subjective phenomena. Nursing Research, 38, 286-288.

Greeneich, D. (1993). The link between new and return business and quality of care: Patient satisfaction. Advances in Nursing Science, 16(1), 62-72.

Heffring, M. P. (1986). High tech, high touch: Common denominators in patient satisfaction. Hospital and Health Services Administration, 31, 81-93. Hinshaw, A., \& Atwood, J. (1982). A patient satisfaction instrument: Precision by replication. Nursing Research, 3i(3), 170-191.

Huskisson, E. C. (1983). Visual analogue scales. In R. Melzack (Ed.), Pain measurement and assessment (pp. 3337). New York: Raven Press.

Inguanzo, J. M., \& Harju, M. (1985). What's the market for emergency care? Hospitals, 59(11), 53-54. 
Jarrell, S. B. (1994). Basic statistics. Dubuque, IA: William Brown.

Kanar, R. J. (1988). The influence of a quality assurance program on patient satisfaction. Journal of Nursing Quality Assurance, 2 (3), 36-43.

Krishel, S., \& Baraff, L. J. (1993). Effect of emergency department information on patient satisfaction. Annals of Emergency Medicine, 22 (3), 568-572.

Larson, P. (1984). Important nursing care behaviors perceived by patients with cancer. Oncology Nursing Forum, $11(6), 46-50$.

Lele, M. M. (1988, December). Manage customers' expectations to buila satisfaction. Business Marketing, p. 64 .

Lele, M. M., \& Sheth, J. N. (1988, June). The four fundamentals of customer satisfaction. Business Marketing, pp. 80-94.

Lemke, R. W. (1987). Identifying consumer satisfaction through patient surveys. Health Proqress, 12, 56-58. Mangen, S. P., \& Griffith, J. H. (1982). Patient satisfaction with community psychiatric nursing: A prospective controlled study. Journal of Advanced Nursing, $\underline{7}, 477-482$. 
McMillan, J. R., Younger, M. S., \& Dewine, L. C. (1986). Satisfaction with hospital emergency department as a function of triage. HCM Review, 11(3), 21-27.

Miller, S., \& Grant, R. (1979). The blunting hypothesis: A view of predictability and human stress. In Trends in behavior therapy (pp. 135-151). New York: Academic Press.

Munro, B. H., Visintainer, M. A., \& Page, E. B. (1986). Statistical methods for healthcare research. Philadelphia: Lippincott.

Oberst, M. (1984). Patients' perceptions of care: Measurement of quality and satisfaction. Cancer, 53, 2366-2373.

O'Connor, S. J. (1988). Service quality, service marketing, and the health care consumer: A study assessing the dimensions of service quality and their influence on patient satisfaction and intention to return (Doctoral dissertation, University of Alabama at Birmingham, 1988). Dissertation Abstracts International, $\underline{49}(12), 5214 \mathrm{~B}$.

Oliver, R. (1980). A cognitive model of the antecedents and consequences of satisfaction decisions. Journal of Marketing Research, 17,460-469. 
Pane, G., Farner, M., \& Salness, K. (1991). Health care access problems of medically indigent emergency department walk-in patients. Annals of Emergency Medicine, $20(7), 730-733$.

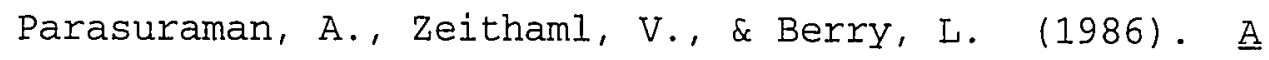
conceptual model of service quality and its implications for further research (Report No. 84-108). Cambridge, MA: Marketing Science Institute.

Pascoe, G., \& Attkisson, C. (1983). The evaluation ranking scale: A new methodology for assessing satisfaction. Evaluation and Program Planning, 6 , 335-347.

Peterson, M. (1991). Patient anxiety before cardiac catheterization: An intervention study. Heart and Lung, 20, 643-647.

Peyrot, M., Cooper, P., \& Schnapf, D. (1993, Winter). Consumer satisfaction and perceived quality of outpatient health care. Journal of Health Care Marketing, $1,24-28$.

Press, I., \& Ganey, R. (1990). Quality of care and patient satisfaction. Quality Letter for Health Care Leaders, $\underline{2}(1), 1-16$.

Raper, J. L. (1994). Patient acuity and patient satisfaction with emergency department nursing care. Unpublished doctoral dissertation, University of Alabama at Birmingham. 
Risser, N. I. (1975). Development of an instrument to measure patient satisfaction with nurses and nursing care in primary care settings. Nursing Research, $\underline{24}(1), 45-52$.

Robinson, B. (1978). A study of consumer perceptions related to nursing. Nursing Leadership, 1(1), 14-18.

Schultz, M. (1986, February). Emergency department utilization--Patient and nurse perceptions. Paper presented at Sigma Theta Tau Research Conference, San Diego.

Sheehy, S. B. (1992). Communicating with patients. In S. B. Sheehy (Ed.), Emergency nursing principles and practice (pp. 19-22). St. Louis: Mosby Year Book. Shesser, R., Kirsch, T., Smith, J., \& Hirsch, R. (1991). An analysis of emergency department use by patients with minor illness. Annals of Emergency Medicine, $\underline{20}(7), 743-748$.

Smeltzer, C. H., \& Curtis, L. (1987). Emergency department care: Many perceptions. Nursing Management, 18(11), $96 \mathrm{~A}-\mathrm{H}$.

Spicer, J. G., Craft, M., \& Ross, K. (1988). A systems approach to customer satisfaction. Nursing Administration Quarterly, $12(3), 79-83$.

SPSS. (1990). SPSS/PC+ computer software. Chicago: Author. 
Swan, J., Sawyer, J., Van Matre, J., \& McGee, A. (1985). Deepening the understanding of hospital patient satisfaction: Fulfillment and equity effects. Journal of Health Care Marketing, $\underline{5}(3), 7-18$.

Swan, J., \& Trawick, I. (1981). Discomfirmation of expectations and satisfaction of a retail service. Journal of Retailing, 57(10), 18-34.

Technical Assistance Research Programs Institute (TRAR). (1986). Consumer complaint handling in America: An update study. Consumer Affairs Council of the U.S. Office of Consumer Affairs. Washington, DC: U.S. Department of Health and Human Services.

Thompson, M. B. (1994). The effects of guided imagery on anxiety levels and movement of clients undergoing magnetic resonance imaging. Holistic Nurse Practice, $\underline{8}(2), 59-69$.

Tilbury, M. S., \& Fisk, T. A. (1989). Marketing and nursing a contemporary view. Owings Mills, MD: National Health Publishing.

Vuori, H. (1987). Patient satisfaction--An attribute or indicator of the quality of care? Quality Review Bulletin, pp. 106-108.

Waltz, C. F., Strickland, O. L., \& Lenz, E. K. (1991). Measurement in nursing research (2nd ed.). Philadelphia: F. A. Davis Co. 
Ware, J., Davies-Avery, A., \& Steward, A. (1978). The measurement and meaning of patient satisfaction. Health \& Medical Care Services Review, 1(1), 1-15. Watkins, L., Weaver, L., \& Odegaard, V. (1986). Preparation for cardiac catheterization: Tailoring the content of instruction to coping style. Heart and Lung, 15, 382-389.

Watson, J. (1979). Nursing: The philosophy and science of caring. Boston: Little, Brown.

Wewers, M., \& Lowe, N. (1990). A critical review of visual analogue scales in the measurement of clinical phenomena. Nursing Research, 13, 227-236.

Wolf, Z. R. (1986). The caring concept and nurse identified caring behaviors. Topics in Clinical Nursing, $\underline{8}(2), 84-93$.

Woodside, A., Frey, L., \& Daly, R. (1989). Linking service quality, customer satisfaction, and behavioral intention. Journal of Health Care Marketing, 9 (4), 517.

Zeithaml, V. A., Parasuraman, A., \& Berry, L. (1990). Delivering quality service: Balancing customer perceptions and expectations. New York: The Free Press. 
APPENDIX A

SAMPLE CONSUMER EMERGENCY CARE SATISFACTION SCALE ITEMS AND SCORING PROCEDURE 


\section{Sample Consumer Emergency Care Satisfaction Scale}

(Davis \& Bush, 1995)

DIRECTIONS: For each statement, indicate how much you agree or disagree with the statement based on this visit to the emergency room by putting an $X$ in the appropriate space. Think of the nurse who spent the most time with you.

EXAMPLE :

Completely

Agree
Completely

Disagree

A. The nurse thought I understood more than I really did.

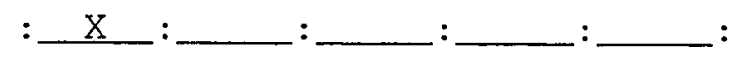

The answer to question $A$ indicates that you are quite certain that the nurse thought you understood more than you really did.
Completely
Agree
Completely
Disagree

1. The nurse was skillful in performing his/her duties.

2. The nurse seemed to be knowledgeable about my illness/problem.

$$
:
$$
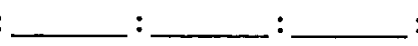

3. The nurse knew what treatment I needed.

4. The nurse gave me instructions about caring for myself at home.

6. The nurse told me what problems to watch for.

7. The nurse told me what to expect at home.

$:$

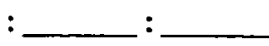
$:$ $:$ : -

$:$ : : : $:$ : : : : :

8. The nurse explained all procedures before they were done. 
Completely Agree

10. The nurse explained things in terms I could understand.

11. The nurse was understanding when listening to my problems.

12. The nurse seemed genuinely concerned about my pain, fear and anxiety.

13. The nurse was as gentle as he/she could be when performing painful procedures.

14. The nurse treated me as a number instead as a person.
Completely

Disagree 


\section{SCORING OF DAVIS CECSS}

The following is the breakdown for the subscales:

Items $1,2,3,4,8,9,11,13,15,16,18$, and 19 comprise the Caring Subscale.

Items 5 through 7 comprise the Teaching Subscale.

Items, 10, 12, 14, and 17 (all negatively worded) are to be considered filler items used to minimize response set and should not be scored.

The scores on each item should range from 1 to 5 with 5 being the most satisfied.

a. Total scale - range possible 15-75 (45)

$<45$ not satisfied

$>45$ satisfied

b. Caring subscale - range possible 12-60 (36)

$<36$ not satisfied

$>36$ satisfied

c. Teaching subscale - range possible 3-15

(9)

$<9$ not satisfied

$>9$ satisfied 
APPENDIX B

LINEAR ANALOGUE ANXIETY SCALE

Reproduced with permission of the copyright owner. Further reproduction prohibited without permission. 


\section{Linear Analoque Anxiety Scale (LAAS)}

DIRECTIONS: Indicate your level of anxiety on (1) arrival to the emergency room and (2) at discharge from the emergency room.

EXAMPLE :

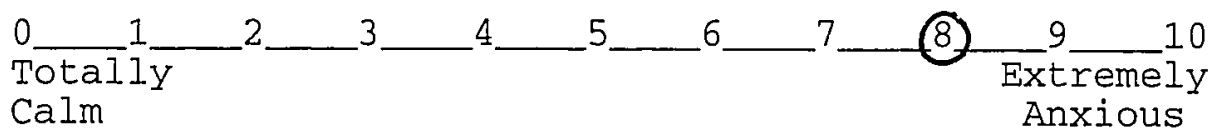

The example indicates a person who felt extremely anxious.

1. Anxiety level on emergency department arrival:

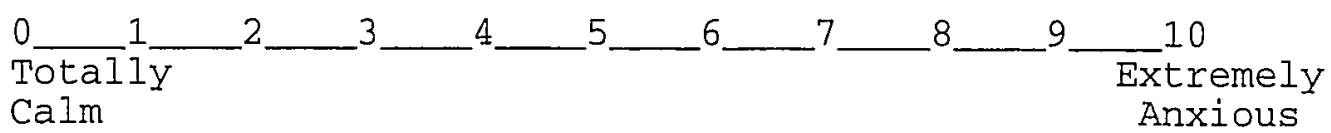

2. Anxiety level on emergency department discharge:

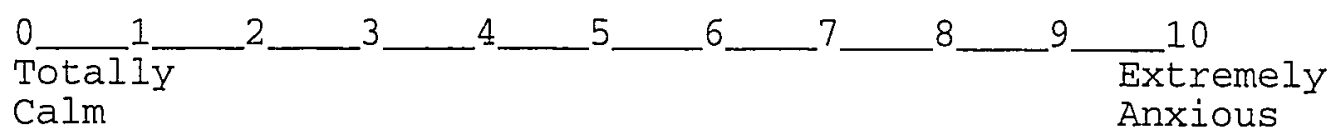


APPENDIX C

SAMPLE INTENT TO RETURN SCALE ITEMS

Reproduced with permission of the copyright owner. Further reproduction prohibited without permission. 
Intent to Return Scale

(Raper, 1994)

Directions: For each statement, indicate on a scale of 1 to 5 how much you agree or disagree with the statement based on this emergency department visit.
Completely
Completely
Agree
Disagree

1. It is possible that in

the future I will use

the same Emergency

Department.

:

:

:

:

:

2. When I next need an Emergency Department, I will return to this Emergency Department.

3. Based on this experience with the Emergency

Department, I would

consider no other

Emergency Departilent in

the future. 
APPENDIX D

WRITTEN INFORMATION SHEET

113

Reproduced with permission of the copyright owner. Further reproduction prohibited without permission. 
Emergency Room

INFORMATION FOR PATIENTS AND VISITORS

Dear Patient/Visitor:

The Emergency Department (E.D.) is a specialized area of the hospital designed to care for patients suffering from acute medical problems. For health reasons, these patients cannot wait to be seen by a private physician. Specially-trained doctors and nurses are available in the E.D. to resolve medical problems by rendering efficient, quality patient care.

Upon your arrival you will be greeted by a Registered Nurse. The nurse will ask you questions to perform an initial evaluation. The information that the nurse obtains will then be passed along to nurses and physicians in the main treatment area.

Next you will be seen by a registration clerk. The clerk will begin a record or chart for you to be used by the E.D. Staff.

When it is your turn to be seen by the doctor, one of the nurses will call you into the treatment area.

Occasionally, there are unavoidable delays and you will not be seen immediately. There are many reasons for delays and why other patients are treated before you. An understanding of these will make your wait less stressful.

\section{EXAMPLES:}

1. Patients may arrive by way of an ambulance at an entrance you may not see. If the medical needs of these patients are more critical than those of the patients in the lobby, the ambulance patient must be seen first.

2. Critical patients, regardless of how they arrive at the E.D., will always be given priority.

3. Some patients come to the E.D. to meet their private physicians for a scheduled appointment. They may be brought back to the treatment area ahead of other patients since they will not be seen by the E.D. staff.

4. Depending on the nature of your problem, it may be necessary to wait for a special room. For example, patients with eye injuries will need to be admitted to an area in which specialized equipment is kept. 
Quality care takes time. You will be under observation by the nursing staff while you wait. Once you have been seen by an E.D. physician it takes time for:

1. Blood work and other test results to come back from the laboratory.

2. X-rays to be processed and results to come back if your $X$-rays were sent to another department to be "read" by a radiologist.

3. Sometimes patients are detained in the E.D. temporarily even after they have received treatment. In this way, the staff can judge the effectiveness of the treatment.

4. If there is a possibility that you need to be admitted, a private physician may need to come see you in the E.D. before you are admitted.

5. Once you have been admitted it takes time to prepare a hospital room to receive you.

\section{VISITORS:}

Please limit visitors to one per patient unless instructed by staff. In this way the patients will have a chance to rest and have the necessary tests performed as quickly as possible. Parents may stay with their children.

Please ask questions. The E.D. staff is here to help you. By understanding the conditions in the E.D. and following these simple rules, you will help the doctors and nurses to provide the best care possible.

\section{BILLING:}

Billing from Sharp Memorial Hospital for your Emergency Room visit will cover the use of the Emergency Room supplies, nursing, technician, pharmacy, lab work, radiology, etc.

A separate bill from the Emergency Room physician will also be sent you. The Emergency Room physician will bill

separately for his/her services. 
APPENDIX E

REASSURANCE DATA COLLECTION FORM 
REASSURANCE DATA COLLECTION FORM

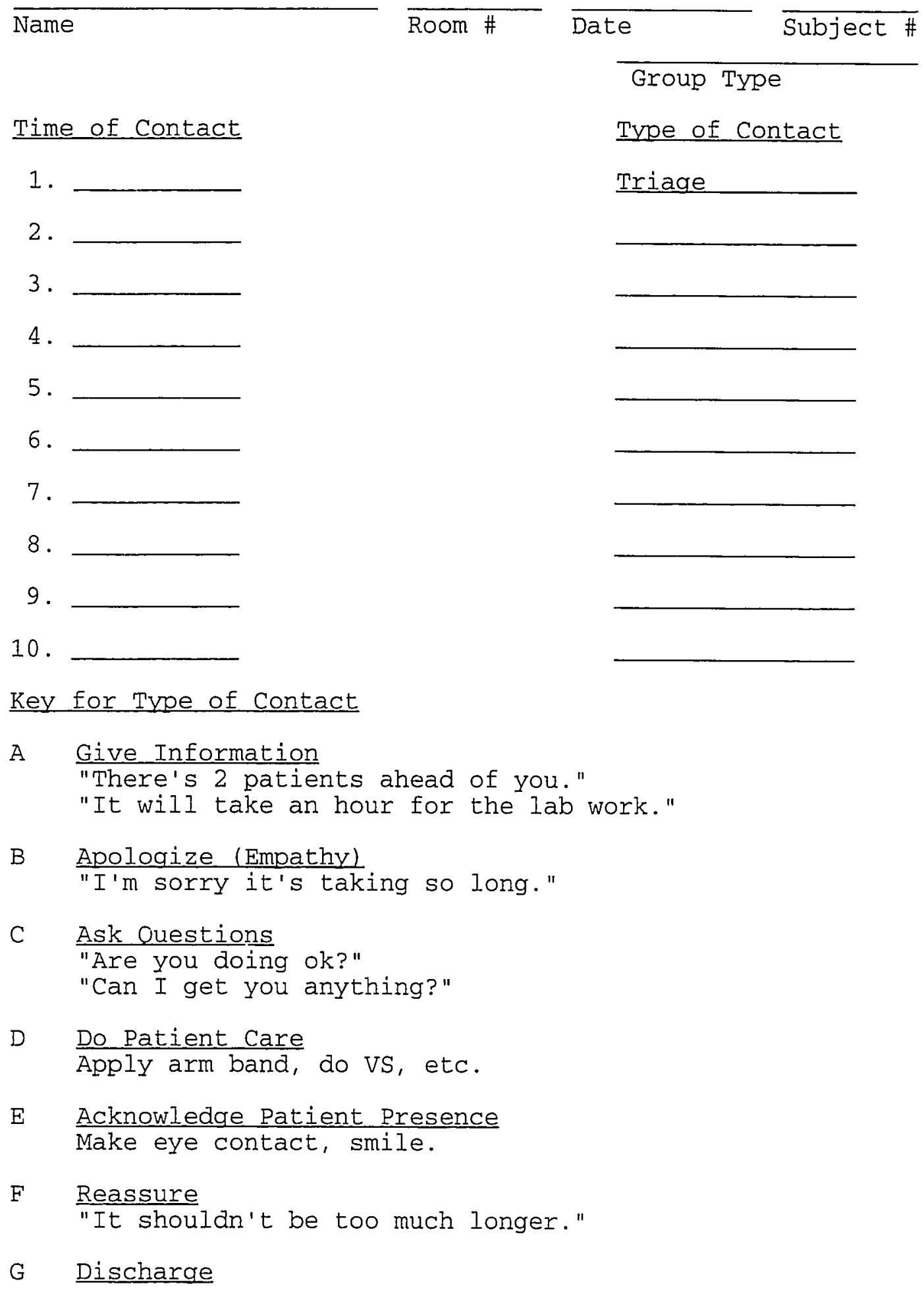


APPEINDIX F

DEMOGRAPHIC DATA COLLECTION FORM

118

Reproduced with permission of the copyright owner. Further reproduction prohibited without permission. 
DEMOGRAPHIC DATA COLLECTION FORM

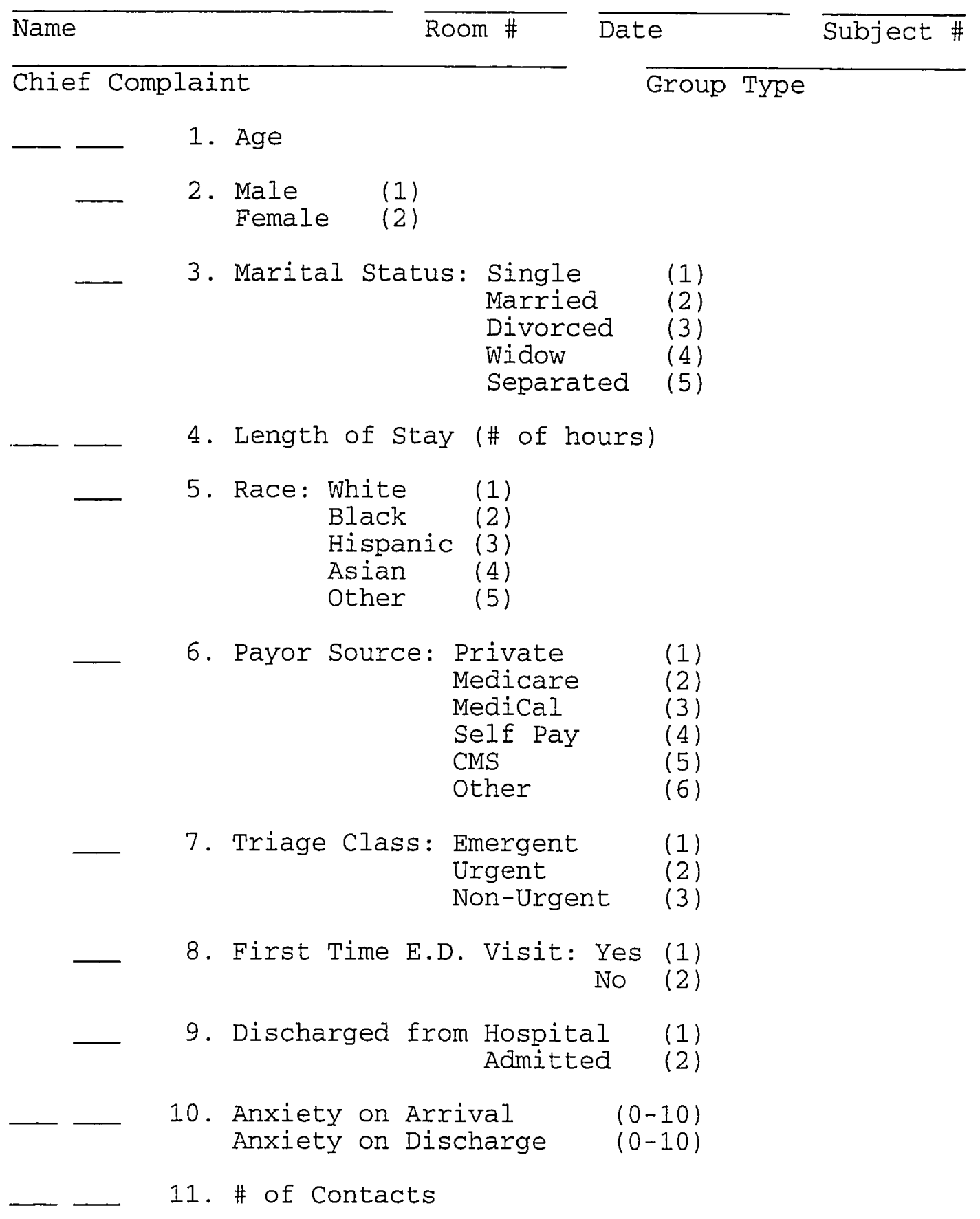


APPENDIX H

SHARP HEALTHCARE INSTITUTIONAL REVIEW BOARD APPROVAL TO CONDUCT RESEARCH 


\section{$\overline{\text { SHARP. }}$ \\ H E A L T H A R E}

May 23, 1995

June Andrea, R.N.

345 Playa Blanca

Encinitas, CA 92024

RE:

The Effect of Written Information and Reassurance on Satient Satisfaction, Anxiety, and Intent to Return for Emergency Care

Dear Ms. Andrea:

This letter is to grant you an EXPEDITED APPROVAL for the study referenced above. This study qualifies as a minimal risk or no risk study under Federal Regulations. I have reviewed your protocol in detail and the informed consent, as well as each of the questionnaires and data collection tools to be utilized in this study. I will report this action to the Institutional Review Board at their June 21, 1995 meeting. Your consent form has also been approved. A copy of the approved informed consent is enclosed with this letter.

If you should have any changes, amendments or revisions to the protocol, please be sure to submit to the IRB office no later than nine working days before the third Wednesday of each month. This will allow such items to be placed on the agenda in a timely fashion and prevent interruption in your study.

If you have any questions, or if I can be of further assistance, please feel free to contact me.

Sincerelv

Laūrence Bavrot, M.D.

Chairman, Institutional Review Board

Sharp HealthCare

LF/gmv

(619) $541-4525$ - 8010 Frost Street - Suite 200 - San Diego, California 92129 


\section{APPENDIX I}

PATIENT CONSENT TO PARTICIPATE IN STUDY

Reproduced with permission of the copyright owner. Further reproduction prohibited without permission. 


\section{University of San Diego and \\ Sharp Memorial Hospital}

CONSENT TO PARTICIPATION IN AN INVESTIGATIONAL STUDY

June Andrea, R.N., DNSc Candidate at the University of San Diego, is conducting a research study to increase understanding of nursing care in the emergency department. I have been asked to take part in this study because I have utilized the emergency department. This research project is conducted under the general guidelines of Sharp Memorial

Hospital, San Diego, California and the guidelines of the University of San Diego.

If I agree to participate in the study, I will be asked to complete a two questionnaires regarding emergency care which will take approximately 15 minutes.

I understand that I may not benefit from the study personally, but the new knowledge gained will help the investigator to better understand ways to improve nursing care in the emergency department. Participation in this study should not involve any added risks or discomforts to me except for possible fatigue or minor psychological distress.

I acknowledge that, before signing this consent, June Andrea or Melissa Dennis has explained this study to me and answered my questions. If I have other questions or research-related problems, I may reach June Andrea, a student at the University of San Diego, at either 541-3291 or 632-9099. An impartial third party, not associated with the study, to whom I may address complaints about the project, is:

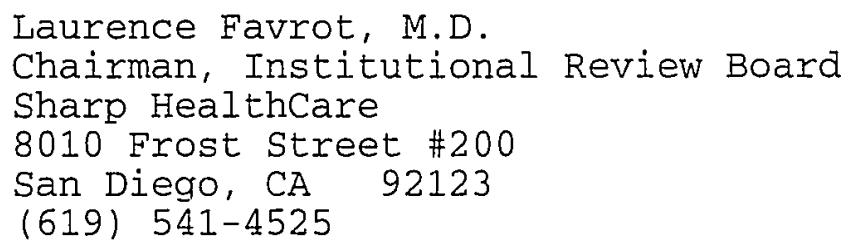

Participation in this research is entirely voluntary. I may refuse to participate or withdraw at any time without jeopardy to any medical care I may receive at Sharp Memorial Hospital. I understand that should I become injured as a result of my participation in this research study, that sharp Memorial Hospital will not provide any compensation or benefits. 
Research records will be kept completely confidential. My identity will not be disclosed without my written consent required by law. I further understand that to preserve my anonymity only group data will be analyzed. There are no other agreements, written or verbal, related to this study beyond that expressed on this consent form.

I have received a copy of this written consent form and a copy of the "Experimental Subject's Bill of Rights". I have also dated and signed the receipt at the foot of the attached "Experimental Subject's Bill of Rights".

I, the undersigned, understand the above explanations and, on that basis, I give consent to my voluntary participation in this research.

Signature of Patient

Date

Signature of Witness

Date 